\title{
Seismic waves and earthquakes in a global monolithic model.
}

\author{
Tomáš Roubíček ${ }^{1,2}$
}

${ }^{1}$ Mathematical Institute, Charles University,Sokolovská 83, CZ-18675 Praha 8, Czech Republic

${ }^{2}$ Institute of Thermomechanics, CAS, Dolejškova 5, CZ-18200 Praha 8, Czech Republic

\begin{abstract}
The philosophy that a single "monolithic" model can "asymptotically" replace and couple in a simple elegant way several specialized models relevant on various Earth layers is presented and, in special situations, also rigorously justified. In particular, global seismicity and tectonics is coupled to capture e.g. (here by a simplified model) ruptures of lithospheric faults generating seismic waves which then propagate through the solid-like mantle and inner core both as shear $(\mathrm{S})$ or pressure $(\mathrm{P})$ waves, while S-waves are suppressed in the fluidic outer core and also in the oceans. The "monolithic-type" models have the capacity to describe all the mentioned features globally in a unified way together with corresponding interfacial conditions implicitly involved, only when scaling its parameters appropriately in different Earth's layers. Coupling of seismic waves with seismic sources due to tectonic events is thus an automatic side effect. The global ansatz is here based, rather for an illustration, only on a relatively simple Jeffreys' viscoelastic damageable material at small strains whose various scaling (limits) can lead to Boger's viscoelastic fluid or even to purely elastic (inviscid) fluid. Self-induced gravity field, Coriolis, centrifugal, and tidal forces are counted in our global model, as well. The rigorous mathematical analysis as far as the existence of solutions, convergence of the mentioned scalings, and energy conservation is briefly presented.
\end{abstract}

AMS Classification: 35K51, 35L20, 35Q86, 74J10, 74R20, 74F10, 76N17, 86A15, 86A17.

Key words: waves; global seismicity; tectonic earthquakes; mathematical models; energy conservation; scaling; convergence proofs, existence of weak solutions.

\section{Introduction}

Global geophysical models typically (have to) deal with several very different phenomena and couple various models due to the layered character of our planet Earth as well as our Moon and many other terrestrial planets or other moons. This paper wants to demonstrate (and, in special situations, also rigorously justify) the philosophy that a single "monolithic" model can replace several specialized models coupled together. Such a single model can be easier to implement on computers algorithmically. Of course, computationally, such a monolithic-type model may be not always easier to produce really relevant simulations at computers we have at our disposal nowadays. As purely seismic global 3D models are already treatable as well as their coupling with earthquake source at least locally, cf. e.g. [11, 12, 14, 29] as well as [8 10, 20], respectively, there is a hope that the coupling monolithic approach may become more amenable in future within ever increasing computer efficiency.

The phenomena we have in mind in this paper involve global seismicity and tectonics. In particular the latter involves e.g. ruptures of lithospheric faults generating seismic waves which then propagate through the solid-like silicate mantle and iron-nickel inner core both in shear $(\mathrm{S})$ or pressure $(\mathrm{P})$ modes. In contrast to the P-waves (also called primary or compressional waves), the S-waves (also called secondary waves) are suppressed in the fluidic iron-nickel outer core and also in the water oceans (where P-waves emitted from the earthquakes in the crust may manifest as Tsunami at the end).

Typically, Maxwell-type rheologies are used in geophysical models of solid mantle to capture long-term creep effects up to $10^{5}$ yrs. Sometimes, more attenuation of Kelvin-Voigt type is also involved, which leads to the Jeffreys rheology [17, cf. also [24]. This seems more realistic in particular because it covers (in the limit) also the Kelvin-Voigt model applied to the volumetric strain whereas the pure Maxwell rheology allowing for big creep during long geological periods 
is not a relevant effect in the volumetric part. We therefore take the Jeffreys model as a basic global "monolithic" ansatz and, in various limits in the deviatoric and the volumetric parts, we model different parts of the planet Earth.

Respecting the solid parts of the model, we use the Lagrangian description, i.e. here all equations are formulated terms of displacements rather than velocities, while the reference and the actual space configurations automatically coincide with each other in our small strain (and small displacement) ansatz, which is well relevant in geophysical short-time scales of seismic events.

In the solid-like part, various inelastic processes are considered to model tectonic earthquakes on lithospheric faults together with long healing periods in between them, as well as aseismic slips, and various other phenomena. To this goal, many internal variables may be involved as aging/damage, inelastic strain, porosity, water content, breakage, and temperature, cf. [15, 17]. On the other hand, those sophisticated models are focused on rather local events around the tectonic faults without ambitions to be directly coupled with the global seismicity. Cf. also [23, 26] for models of rupturing lithospheric faults and, in particular, a relation with the popular Dieterich-Ruina rate-and-state friction model. Here, rather for the lucidity of the exposition, we reduce the set of internal variables to only one scalar variable, namely damage/aging, which however has a capacity to trigger a spontaneous rupture (so-called dynamic triggering) with emission of seismic waves and, in a certain simplification, can serve as a seismic source coupled with the overall global model. Also, this simple model already will well illustrate mathematical difficulties related to nonlinearities in the solid parts coupled with linear but possibly hyperbolic fluidic regions.

Let us emphasize that usual models are focused only either on propagation of seismic waves along the whole globe while their source is considered given, or on description of seismic sources due to tectonic events, but not their mutual coupling. If a coupling is considered, then is concerns rather local models not considering the layered structure of the whole planet, cf. e.g. [1,2,9,10,16]. The reality ultimately captures very different mechanical properties of different layers of the Earth, in particular the mantle and the inner core which are solid from the short-time scales versus the outer core and the oceans which are fluidic even on the short-time scales.

The goals of this article are:

$\alpha$ ) to propose a model that might capture simultaneously propagation of seismic waves over the whole planet and their nonlinearly behaved sources (like ruptures of tectonic faults, here modelled only in a very simplified way for a relatively lucid illustration of the model procedure), both mutually coupled.

ß) by proper scaling to approximate viscoelastic Boger-type [3] fluids that is relevant in outer core and in the oceans (with a very low viscosity) where S-waves can then only slightly penetrate the outer core or the oceans but are fast attenuated, while P-waves are only refracted.

$\gamma$ ) by limiting further the viscosity in the outer core or the oceans to zero, further to approximate this viscoelastic fluid towards elastic (completely inviscid) fluid, respecting the phenomenon that $\mathrm{S}$-waves cannot penetrate into these fluidic regions and are fully reflected on the interfaces between the outer core and the mantle (=Gutenberg's discontinuity) and the inner core or on the ocean beds, i.e. between $\Omega_{\mathrm{S}}$ and $\Omega_{\mathrm{F}}$, while P-waves propagate through these interfaces, being both refracted and reflected on them.

$\delta)$ perform the rigorous analysis as far as the existence of solutions, a-priori estimates in specific norms, and convergence towards other models that justifies the particular models, their energetics and asymptotics, and can support numerical stability and convergence when discretised and implemented on computers.

The plan of this article is the following: In Section 2, the general monolithic model is introduced and also specialized for viscoelastic linear isotropic material undergoing possibly damage in the elastic shear part. In Sections 3 and 4 , we formulate the limit toward viscoelastic and purely elastic fluids, respectively. This limit concerns only the sudbomain $\Omega_{\mathrm{F}}$, i.e. the outer core and 


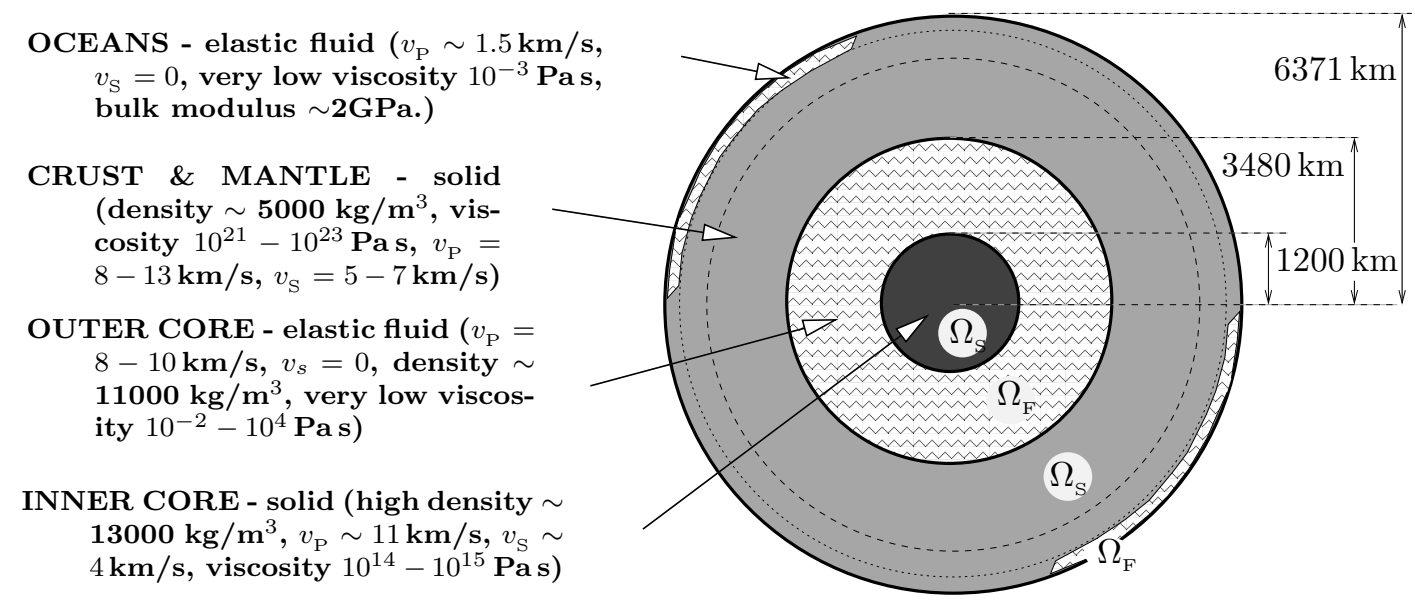

Figure 1: The very basic layered structure of our planet Earth with some (only very rounded) geometrical and rheological data and the notation for the solid-type domains $\Omega_{\mathrm{S}}$ and the fluidtype domains $\Omega_{\mathrm{F}}$. Here, $v_{\mathrm{P}}$ and $v_{\mathrm{S}}$ stand for the velocity of the $P$ - and $S$-waves, respectively, $v_{\mathrm{S}}=0$ indicating that only the $P$ - but not $S$-waves can propagate through the particular layer.

oceans. To make the presentation more lucid and readable also for the geophysical specialist outside mathematical-analyst community, the mathematical analysis of the models and necessarily a bit technical proofs of the statements formulated in Sections 24 are intentionally presented as Appendix later in Section 5. For it, we use a conceptually constructive approximation by the Galerkin method, which is in some variant also used in geophysical literature, cf. e.g. 20].

\section{Philosophy and an example of a monolithic model}

Rather for illustration of our main focus to coupling of solid and fluidic models, we ignore most of the above mentioned internal variables and keep only the scalar-value aging/damage variable, denoted by $\alpha$, and then we consider the mentioned Jeffreys rheology combined with damage influencing only the elastic but not viscous part. The differential relation governing this rheology is of the type " $\dot{\sigma}+\sigma=\ddot{e}+\dot{e} "$ with some specific coefficients (here ignored), some of them being possibly subject to damage. Here and in what follows, the dot-notation $(\cdot)^{*}$ stands for the time derivative $\frac{\partial}{\partial t}$. Alternatively, to make the damage dependence more lucid, one can use system of first-order equations in time when implementing the concept of internal variables, i.e. here introducing a Maxwellian-type creep strain $\pi$ and making a Green-Naghdi [7] additive decomposition of the total strain

$$
e(u)=e_{\mathrm{el}}+\pi
$$

So, considering also the damage/aging variable, altogether our set of internal variables will be $(\pi, \alpha)$.

The main ingredients of the model are then, beside the mass density $\varrho=\varrho(x)$, the specific stored energy and the dissipation potential as a property of the material

$$
\begin{aligned}
& \varphi_{\mathrm{M}}=\varphi_{\mathrm{M}}\left(x, u, e_{\mathrm{el}}, \alpha, \nabla \alpha, \phi, \nabla \phi\right), \\
& \zeta_{\mathrm{M}}=\zeta_{\mathrm{M}}\left(x, \dot{e}_{\mathrm{el}}, \dot{\pi}, \dot{\alpha}\right) .
\end{aligned}
$$

Note that $\varphi_{\mathrm{M}}$ and $\zeta_{\mathrm{M}}$ naturally do not explicitly depend on the total strain $e(u)$ but rather on the elastic strain $e_{\mathrm{el}}$ and possibly on the creep strain $\pi$ and their rates; in fact, $\varphi_{\mathrm{M}}$ is independent of $\pi$ because naturally no hardening-like effects are relevant in geophysical models. In what follows, we will often omit the explicit dependence on $x$ variable.

For readers' convenience, Table 1 summarizes the main nomenclature used in this paper.

To formulate the equations for the dynamics of displacement $u$, it is more natural in the Kelvin-Voigt model (which is a part of the Jeffreys rheology) to express the stored-energy and 
$u$ displacement, valued in $\mathbb{R}^{3}$,

$\pi$ creep strain, valued in $\mathbb{R}_{\mathrm{sym}}^{3 \times 3}$,

$\alpha$ damage/aging, valued in $[0,1]$,

$\phi$ potential of the gravity field,

$e(u)=\frac{1}{2} \nabla u^{\top}+\frac{1}{2} \nabla u$ total strain,

$e_{\mathrm{el}}=e(u)-\pi$ elastic strain,

$\varphi_{\mathrm{M}}, \varphi$ stored energies,

$\zeta_{\mathrm{M}}, \zeta$ potentials of dissipative forces,

$\eta$ potential of damage dissipation,

$f_{\mathrm{COR}}(\dot{u})=2 \varrho \omega \times \dot{u}$ Coriolis force,

$\mathbb{R}_{\mathrm{sym}}^{3 \times 3}=\left\{A \in \mathbb{R}^{3 \times 3} ; A^{\top}=A\right\}$,

$\mathbb{R}_{\mathrm{dev}}^{3 \times 3}=\left\{A \in \mathbb{R}_{\mathrm{sym}}^{3 \times 3} ; \operatorname{tr} A=0\right\}$,

$\mathbb{R}_{\mathrm{sph}}^{3 \times 3}=\left\{A \in \mathbb{R}_{\mathrm{sym}}^{3 \times 3} ; A=a \mathbb{I}, a \in \mathbb{R}\right\}$, $\varrho$ given mass density profile,

$\varrho_{\text {ext }}$ external mass causing tidal forces,

$K_{\mathrm{E}}, G_{\mathrm{E}}$ bulk and shear elastic moduli,

$K_{\mathrm{MX}}, G_{\mathrm{Mx}}$ Maxwell viscous moduli,

$K_{\mathrm{KV}}, G_{\mathrm{KV}}$ Kelvin-Voigt viscous moduli,

$g$ the gravitational constant

$\omega$ given angular velocity of Earth rotation,

$\Omega \subset \mathbb{R}^{3}$ the reference domain (the Earth),

$\Omega_{\mathrm{F}} \subset \Omega$ the outer core and the oceans,

$\Omega_{\mathrm{S}} \subset \Omega$ the mantle and the inner core,

$\Gamma$ the boundary of $\Omega$ (=Earth surface),

$I:=[0, T]$ the fixed time interval,

$Q:=I \times \Omega, Q_{\mathrm{S}}:=I \times \Omega_{\mathrm{S}}, Q_{\mathrm{F}}:=I \times \Omega_{\mathrm{F}}$,

$\Sigma:=I \times \Gamma$.

Table 1. Summary of the basic notation used through the paper.

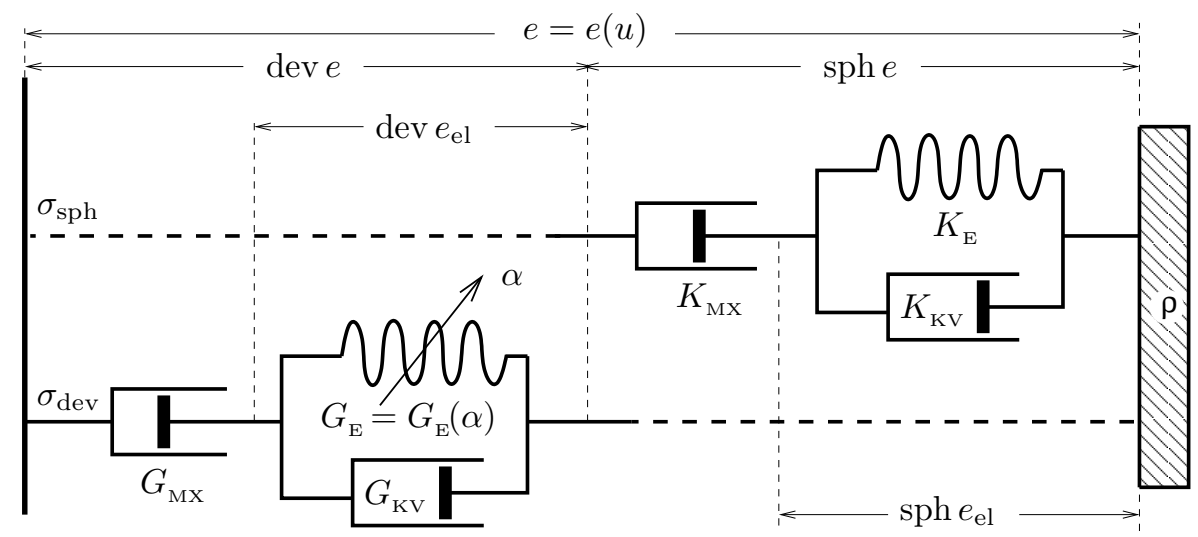

Figure 2: Schematic diagram for the viscoelastic Jeffreys rheology which is subjected to damage $\alpha$ in the deviatoric part while the spherical (volumetric) part is not subjected to damage.

dissipation potentials rather in terms of the total strain $e=e(u)$ and the creep $\pi$ from (2.1) as

$$
\begin{aligned}
& \varphi(x, u, e, \pi, \alpha, \nabla \alpha, \phi, \nabla \phi)=\varphi_{\mathrm{M}}(x, u, e-\pi, \alpha, \nabla \alpha, \phi, \nabla \phi) \\
& \zeta(\dot{e}, \dot{\pi}, \dot{\alpha})=\zeta_{\mathrm{M}}(\dot{e}-\dot{\pi}, \dot{\pi}, \dot{\alpha}) .
\end{aligned}
$$

In terms of these potentials, our system in the abstract form is:

$$
\begin{aligned}
\varrho \ddot{u}+\varphi_{u}^{\prime}(\nabla \phi)-\operatorname{div}\left(\zeta_{\dot{e}}^{\prime}(e(\dot{u}), \dot{\pi})+\varphi_{e}^{\prime}(e(u), \pi, \alpha)\right) & =f_{\mathrm{COR}}(\dot{u}) & & \text { in } \Omega, \\
\zeta_{\dot{\pi}}^{\prime}(e(\dot{u}), \dot{\pi})+\varphi_{\pi}^{\prime}(e(u), \pi, \alpha) & =0 & & \text { in } \Omega, \\
\zeta_{\alpha}^{\prime}(\dot{\alpha})+\varphi_{\alpha}^{\prime}(e(u), \pi, \alpha)-\operatorname{div}\left(\varphi_{\nabla \alpha}^{\prime}(\nabla \alpha)\right) & =0 & & \text { in } \Omega, \\
\varphi_{\phi}^{\prime}(u, \phi)-\operatorname{div}\left(\varphi_{\nabla \phi}^{\prime}(\nabla \phi)\right) & =\varrho_{\mathrm{ext}}(t) & & \text { in } \mathbb{R}^{3},
\end{aligned}
$$

where $(\cdot)^{\prime}$ denotes the (partial) derivatives for which we already have assumed a certain particular form of (2.2) so that the partial derivatives of $\varphi$ and $\zeta$ do not depend on the full list of arguments, thus e.g. instead of $\varphi_{e}^{\prime}(u, e(u), \pi, \alpha, \nabla \alpha, \phi, \nabla \phi)$ we wrote only $\varphi_{e}^{\prime}(e(u), \pi, \alpha)$, etc. Note that, in (2.4a), we used also a bulk force $f_{\mathrm{COR}}(\dot{u})$ which may not come from the dissipation potential by a classical way $f_{\mathrm{COR}}(\dot{u})=\zeta_{\dot{u}}$, which in particular the case of the Coriolis (pseudo) force which itself does not vanish but nevertheless the dissipation rate $f_{\mathrm{COR}}(\dot{u}) \cdot \dot{u}=0$.

Rheological response under the volume variation and under the shear may (and do) substantially differ from each other. To distinguish these geometrical aspects, the total strain is decomposed to the spherical (also called hydrostatic or volumetric) strain and the deviatoric 
(also called shear) strain:

$$
e=\operatorname{sph} e+\operatorname{dev} e \quad \text { with } \quad \operatorname{sph} e=\frac{\operatorname{tr} e}{3} \mathbb{I} \quad \text { and } \quad \operatorname{dev} e=e-\frac{\operatorname{tr} e}{3} \mathbb{I},
$$

where $\mathbb{I}$ is the identity matrix and "tr" denotes the trace of a matrix. Note that the deviatoric and the spherical strains from (2.5) are orthogonal to each other. In terms of this decomposition, for example the isotropic elastic (Lamé) material at small strains has the quadratic stored energy

$$
\begin{aligned}
\varphi_{\text {Lamé }}(e) & =\frac{1}{2} \lambda(\operatorname{tr} e)^{2}+G_{\mathrm{E}}|e|^{2}=\frac{1}{2}\left(\lambda+\frac{2}{3} G_{\mathrm{E}}\right)(\operatorname{tr} e)^{2}+G_{\mathrm{E}}\left|e-\frac{\operatorname{tr} e}{3} \mathbb{I}\right|^{2} \\
& =\frac{1}{2}\left(3 \lambda+2 G_{\mathrm{E}}\right)|\operatorname{sph} e|^{2}+G_{\mathrm{E}}|\operatorname{dev} e|^{2} \\
& =\frac{3}{2} K_{\mathrm{E}}|\operatorname{sph} e|^{2}+G_{\mathrm{E}}|\operatorname{dev} e|^{2} \quad \text { with } K_{\mathrm{E}}=\lambda+\frac{2}{3} G_{\mathrm{E}} .
\end{aligned}
$$

where we used $|\operatorname{sph} e|^{2}=\left(\frac{\operatorname{tr} e}{3}\right)^{2} \mathbb{I}: \mathbb{I}=\left(\frac{\operatorname{tr} e}{3}\right)^{2} 3=(\operatorname{tr} e)^{2} / 3$ and the mentioned orthogonality of sph $e$ and $\operatorname{dev} e$, where $K_{\mathrm{E}}$ is called the bulk modulus and $\lambda$ is the (first) Lamé coefficient while $G_{\mathrm{E}}$ is the shear modulus (= the second Lamé coefficient). Then the corresponding stress writes as:

$$
\begin{aligned}
\varphi_{\text {Lamé }}^{\prime}(e)= & \lambda(\operatorname{tr} e) \mathbb{I}+2 G_{\mathrm{E}} e=: \sigma_{\mathrm{dev}}+\sigma_{\mathrm{sph}} \\
& \text { with } \sigma_{\mathrm{dev}}=2 G_{\mathrm{E}} \operatorname{dev} e \text { and } \sigma_{\mathrm{sph}}=3 K_{\mathrm{E}} \mathrm{sph} e .
\end{aligned}
$$

We will use this decomposition both for elastic and for the viscous parts of our model and let the elastic bulk modulus $G_{\mathrm{E}}$ and thus also $\varphi$ dependent on damage. Yet, this dependence would bring mathematical difficulties (like lack of the integrability of the term $\dot{\alpha} G_{E}(\alpha)\left|\operatorname{dev} e_{\mathrm{el}}\right|^{2}$ that would arise in (2.11), for example). Here, various modifications of the Lamé-type model can help. One option is to consider higher strain gradients, i.e. the concept of nonsimple continua, cf. also [24]. Other (conceptually and technically simpler) option is to modify the stored energy by considering an non-quadratic $e \mapsto \mathcal{G}_{\mathrm{E}}(\alpha, \operatorname{dev} e)$ instead of $e \mapsto G_{E}(\alpha)|\operatorname{dev} e|^{2}$. A canonical option one may have in mind is

$$
\mathcal{G}_{\mathrm{E}}\left(\alpha, e_{\mathrm{el}}\right)=\mathcal{G}_{\mathrm{E}}\left(\alpha, \operatorname{dev} e_{\mathrm{el}}\right)=\frac{G_{\mathrm{E}}(\alpha)\left|\operatorname{dev} e_{\mathrm{el}}\right|^{2}}{\sqrt{1+\left|\operatorname{dev} e_{\mathrm{el}}\right|^{2} / E_{\mathrm{M}}^{2}}}
$$

for (presumably large) regularizing parameter $E_{\mathrm{M}}$. In accord with Figure 2, in (2.7) and in what follows, we quite naturally assume that $\mathcal{G}_{\mathrm{E}}$ does not depend on the spherical part of the strain which itself usually cannot make any damage in the rock. The meaning of $E_{\mathrm{M}}$ in (2.7) is clear from realizing that, if the deviatoric strain is substantially smaller in the sense $\left|\operatorname{dev} e_{\mathrm{el}}\right|<<E_{\mathrm{M}}$, then the difference from the original Lameé-type model is negligible, i.e. the corresponding stress contribution $\partial_{e} \mathcal{G}_{\mathrm{E}}\left(\alpha, e_{\mathrm{el}}\right) \sim 2 G_{\mathrm{E}}(\alpha) \operatorname{dev} e_{\mathrm{el}}$. On the other hand, this modification is effective for large strains and makes $e_{\mathrm{el}} \mapsto \varphi_{\mathrm{M}}\left(x, u, e_{\mathrm{el}}, \alpha, \nabla \alpha, \phi, \nabla \phi\right)$ nonquadratic but still keeps convex now with at most linear growth, so that the elastic part of deviatoric stress is always below $G_{\mathrm{E}}(\alpha) E_{\mathrm{M}}$. Thus, for $E_{\mathrm{M}}$ large, this is effectively not a substantial (and physically well acceptable) modification of the original model.

Thus we consider the material stored energy and the material dissipation-force potential from (2.2) governing the problem as

$$
\begin{gathered}
\varphi_{\mathrm{M}}\left\{\begin{array}{cc}
=\varphi_{\mathrm{M}}\left(x, u, e_{\mathrm{el}}, \alpha, \nabla \alpha, \phi, \nabla \phi\right)=\frac{3}{2} K_{\mathrm{E}}\left|\operatorname{sph} e_{\mathrm{el}}\right|^{2}+\mathcal{G}_{\mathrm{E}}\left(\alpha, \operatorname{dev} e_{\mathrm{el}}\right) \\
+\frac{\kappa}{2}|\nabla \alpha|^{2}+\varrho \phi+\varrho \nabla \phi \cdot u+\frac{1}{8 \pi g}|\nabla \phi|^{2} \\
\quad+\frac{1}{2} \varrho\left(|\omega \cdot(x+u)|^{2}-|\omega|^{2}|x+u|^{2}\right) & \text { if } x \in \Omega, \\
=\varphi_{\mathrm{M}}(\nabla \phi)=\frac{1}{8 \pi g}|\nabla \phi|^{2} & \text { if } x \in \mathbb{R}^{3} \backslash \Omega,
\end{array}\right. \\
\zeta_{\mathrm{M}}=\zeta_{\mathrm{M}}\left(x, \dot{e}_{\mathrm{el}}, \dot{\pi}, \dot{\alpha}\right)=\frac{3}{2} K_{\mathrm{KV}}\left|\operatorname{sph} \dot{e}_{\mathrm{el}}\right|^{2}+G_{\mathrm{KV}}\left|\operatorname{dev} \dot{e}_{\mathrm{el}}\right|^{2} \\
+\frac{3}{2} K_{\mathrm{MX}}|\operatorname{sph} \dot{\pi}|^{2}+G_{\mathrm{MX}}|\operatorname{dev} \dot{\pi}|^{2}+\eta(\dot{\alpha})
\end{gathered}
$$


with $g \doteq 6.674 \times 10^{-11} \mathrm{~m}^{3} \mathrm{~kg}^{-1} \mathrm{~s}^{-2}$ the gravitational constant. For the $\phi$-terms in (2.8a) see e.g. [32, Formulae $(16),(20)]$. All the coefficients $K$ 's, $G$ 's, and $\kappa$ 's are naturally considered defined on $\Omega$ and $x$-dependent (which is not explicitly written in (2.8) for brevity). Also the mass density is considered $x$-dependent and, because of $(2.10 \mathrm{~d})$, defined on the whole Universe $\mathbb{R}^{3}$ by putting $\varrho(x)=0$ if $x \in \mathbb{R}^{3} \backslash \Omega$. Keeping $K_{\mathrm{E}}$ constant in (2.8a) reflects the phenomenon that compression does not cause damage and the pure tension is not a relevant mode in geophysical models, so only shear does cause damage because of dependence of the elastic shear modulus $G_{\mathrm{E}}$ in (2.7) and (2.8a) on $\alpha$.The last term in (2.8a) is the potential of the centrifugal force $\varrho \omega \times(\omega \times(x+u))$ and, noteworthy, it violates coercivity due to the term $-\frac{1}{2} \varrho|\omega|^{2}|x+u|^{2}$, which reflects the real phenomenon that, given the angular velocity $\omega$, the centrifugal force can indeed inflate the body in an unlimited way in the direction orthogonal to the rotation axis. Of course, in reality, this is either not observed in planetary/satellite bodies or the angular velocity cannot be taken apriori fixed in some large-type bodies.

For shorter notation, we define the 4th-order tensors corresponding to the isotropic material expressed in terms of the $(K, G)$-moduli in (2.8), namely

$$
\begin{aligned}
& {[\mathbb{C}(\alpha, e)]_{i j k l}=K_{\mathrm{E}} \delta_{i j} \delta_{k l}+\partial_{e e} \mathcal{G}_{\mathrm{E}}(\alpha, e)\left(\delta_{i k} \delta_{j l}+\delta_{i l} \delta_{j k}-2 \delta_{i j} \delta_{k l} / 3\right),} \\
& {\left[\mathbb{D}_{\mathrm{MX}}\right]_{i j k l}=K_{\mathrm{MX}} \delta_{i j} \delta_{k l}+G_{\mathrm{MX}}\left(\delta_{i k} \delta_{j l}+\delta_{i l} \delta_{j k}-2 \delta_{i j} \delta_{k l} / 3\right),} \\
& {\left[\mathbb{D}_{\mathrm{KV}}\right]_{i j k l}=K_{\mathrm{KV}} \delta_{i j} \delta_{k l}+G_{\mathrm{KV}}\left(\delta_{i k} \delta_{j l}+\delta_{i l} \delta_{j k}-2 \delta_{i j} \delta_{k l} / 3\right),}
\end{aligned}
$$

where $\delta$ 's denote the Kronecker symbol. In what follows, we will take specifically $f_{\mathrm{COR}}(\dot{u})=$ $2 \varrho \omega \times \dot{u}$ which is the standard Coriolis force with $\omega$ the given vector (angular velocity related with the constant rotation of the Earth with respect to the inertial system). The general system (2.4) then takes the more specific form:

$$
\begin{aligned}
& \varrho \ddot{u}-\operatorname{div} \sigma+f=0 \quad \text { with } \sigma=\mathbb{D}_{\mathrm{KV}} \dot{e}_{\mathrm{el}}+\mathbb{C}\left(\alpha, \operatorname{dev} e_{\mathrm{el}}\right) e_{\mathrm{el}} \\
& \text { and } f=\varrho(\omega \times(\omega \times(x+u))+2 \omega \times \dot{u}+\nabla \phi) \quad \text { in } \Omega \text {, } \\
& \mathbb{D}_{\mathrm{Mx}} \dot{\pi}=\sigma \\
& \partial \eta(\dot{\alpha})+\partial_{\alpha} \mathcal{G}_{\mathrm{E}}\left(\alpha, \operatorname{dev} e_{\mathrm{el}}\right)-\operatorname{div}(\kappa \nabla \alpha) \ni 0 \\
& \operatorname{div}\left(\frac{1}{4 \pi g} \nabla \phi+\varrho u\right)=\varrho+\varrho_{\text {ext }}(t)
\end{aligned}
$$

The bulk force $f$ in (2.4a) involves the centrifugal force, the Coriolis force, and the gravity force due to the self-induced gravity field. This last force plays a certain role in ultra-low frequency (i.e. very long wavelength) seismic waves, cf. e.g. [29]. For such right-hand side in (2.10a) together with (2.10d) see e.g. [32, Formulae (19),(22)]. Actually, this self-gravity interaction results after a certain linearization of the system originally written at large strains, cf. also [4,5]. The external time-varying mass density $\varrho_{\text {ext }}=\varrho_{\text {ext }}(x, t)$ occurring in (2.10d $)$ allows for involvement of tidal forces arising from other bodies (stars, planets, moons) moving around. The notation $\partial \eta$ in (2.10c) stands for a subdifferential of the convex function $\eta$ which standardly generalizes the usual derivative if $\eta$ is non-smooth and, by definition, the inclusion " $\ni$ " there actually means, for any $v \in \mathbb{R}$ and a.a. $(t, x) \in Q$, the inequality

$$
(v-\dot{\alpha}(t, x))\left(\partial_{\alpha} \mathcal{G}_{\mathrm{E}}\left(x, \alpha(t, x), \operatorname{dev} e_{\mathrm{el}}(t, x)\right)-\operatorname{div}(\kappa \nabla \alpha(t, x))\right)+\eta(v) \geq \eta(\dot{\alpha}(t, x)) .
$$

To facilitate spontaneous rupture on some weak narrow layers (pre-existing faults in the solid crust) that leads to earthquakes, some "enough pronounced" nonconvexity of $\varphi$ is necessary. One option was devised in [18, introducing non-convexity in terms of $e_{\mathrm{el}}$ if damage develops enough, and used in a series of papers, see e.g. [15, 17] and references therein. Mathematically rigorous setting of this model requires however some higher-order strain gradients (the so-called 2ndgrade nonsimple materials) and is rather technical, cf. 24]. Alternative nonconvexity can be implemented through $\mathcal{G}_{\mathrm{E}}(\cdot, e)$ causing possible weakening when damage develops, being opposite to so-called cohesive damage. To avoid the mentioned mathematical technicalities, we have chosen the latter option. 
To see the energetics behind the system (2.4), we test the particular equations in (2.4) by $\dot{u}$, $\dot{\pi}, \dot{\alpha}$, and $\dot{\phi}$, respectively. Thus we obtain (at least formally) the energy balance

$$
\begin{aligned}
\frac{\mathrm{d}}{\mathrm{d} t}\left(\int_{\Omega} \frac{\varrho}{2}|\dot{u}|^{2} \mathrm{~d} x+\int_{\mathbb{R}^{3}} \varphi(u, e(u), \pi, \alpha, \nabla \alpha, \phi, \nabla \phi) \mathrm{d} x\right) \\
+\int_{\Omega} \xi(e(\dot{u}), \dot{\pi}, \dot{\alpha}) \mathrm{d} x=\int_{\mathbb{R}^{3}} \varrho_{\operatorname{ext}}(t) \dot{\phi} \mathrm{d} x,
\end{aligned}
$$

where $\xi=\xi(\dot{e}, \dot{\pi}, \dot{\alpha})$ is the dissipation rate related with the dissipation potential $\zeta=\zeta(\dot{e}, \dot{\pi}, \dot{\alpha})$ by $\xi(\dot{e}, \dot{\pi}, \dot{\alpha})=(\dot{e}, \dot{\pi}, \dot{\alpha})^{\top} \partial \xi(\dot{e}, \dot{\pi}, \dot{\alpha})$. Using (2.8) and (2.9), we can make it more specific for the system (2.10) when using also the calculus

$$
\int_{\Omega} \varrho \nabla \phi \cdot \dot{u}-\operatorname{div}(\varrho u) \dot{\phi} \mathrm{d} x=\int_{\Omega} \varrho \nabla \phi \cdot \dot{u}+\varrho u \cdot \nabla \dot{\phi} \mathrm{d} x=\frac{\mathrm{d}}{\mathrm{d} t} \int_{\Omega} \varrho \nabla \phi \cdot u \mathrm{~d} x .
$$

and realizing that, in view of $(2.3 \mathrm{~b})$ and $(2.8 \mathrm{~b})$,

$$
\begin{aligned}
\xi(\dot{e}, \dot{\pi}, \dot{\alpha})= & 3 K_{\mathrm{KV}}|\operatorname{sph}(\dot{e}-\dot{\pi})|^{2}+2 G_{\mathrm{KV}}|\operatorname{dev}(\dot{e}-\dot{\pi})|^{2} \\
& +3 K_{\mathrm{MX}}|\operatorname{sph} \dot{\pi}|^{2}+2 G_{\mathrm{MX}}|\operatorname{dev} \dot{\pi}|^{2}+\dot{\alpha} \eta^{\prime}(\dot{\alpha}) \\
= & \mathbb{D}_{\mathrm{KV}}(\dot{e}-\dot{\pi}):(\dot{e}-\dot{\pi})+\mathbb{D}_{\mathrm{MX}} \dot{\pi}: \dot{\pi}+\dot{\alpha} \eta^{\prime}(\dot{\alpha})
\end{aligned}
$$

when using also the notation (2.9), $\mathrm{c})$. Thus (2.12) reads more speficially here as

$$
\begin{aligned}
& \frac{\mathrm{d}}{\mathrm{d} t}\left(\int_{\Omega} \frac{\varrho}{2}|\dot{u}|^{2}+\frac{3}{2} K_{\mathrm{E}}|\operatorname{sph}(e(u)-\pi)|^{2}+\mathcal{G}_{\mathrm{E}}(\alpha, \operatorname{dev}(e(u)-\pi))+\varrho \phi+\varrho \nabla \phi \cdot u\right. \\
& \left.+\frac{\kappa}{2}|\nabla \alpha|^{2}+\frac{1}{2} \varrho\left(|\omega \cdot(x+u)|^{2}-|\omega|^{2}|x+u|^{2}\right) \mathrm{d} x+\int_{\mathbb{R}^{3}} \frac{1}{8 \pi g}|\nabla \phi|^{2} \mathrm{~d} x\right) \\
& +\int_{\Omega} \mathbb{D}_{\mathrm{KV}}(e(\dot{u})-\dot{\pi}):(e(\dot{u})-\dot{\pi})+\mathbb{D}_{\mathrm{MX}} \dot{\pi}: \dot{\pi}+\dot{\alpha} \partial \eta(\dot{\alpha}) \mathrm{d} x=\int_{\mathbb{R}^{3}} \varrho_{\operatorname{ext}}(t) \dot{\phi} \mathrm{d} x .
\end{aligned}
$$

Here one should naturally assume that $\eta$ is (naturally) nonsmooth possibly only at $\dot{\alpha}=0$ so that the dissipation rate $\dot{\alpha} \partial \eta(\dot{\alpha})$ is actually a single-valued function. The Coriolis force does not occur in (2.15) because $\omega \times \dot{u}$ is always orthogonal to $\dot{u}$ so that $(\omega \times \dot{u}) \cdot \dot{u}=0$. This is a well known effect that the Coriolis (pseudo) force does not make any work.

Denoting $\vec{n}$ the (unit) normal to the boundary $\partial \Omega$ of $\Omega$, i.e. the Earth surface, we complete the system by natural initial and boundary conditions:

$$
\begin{array}{ll}
\left.u\right|_{t=0}=u_{0},\left.\quad \dot{u}\right|_{t=0}=v_{0},\left.\quad \pi\right|_{t=0}=\pi_{0},\left.\quad \alpha\right|_{t=0}=\alpha_{0} . \\
\sigma \vec{n}=0 \quad \text { on } \partial \Omega, \quad \phi(\infty)=0, \quad \text { and } \\
(\kappa \nabla \alpha) \cdot \vec{n}=0 \text { on } \partial \Omega,
\end{array}
$$

Actually, it would make a good sense to consider also some period instead of initial conditions, but the analysis of such a problem would be more difficult.

It is a conventional modelling property that $\alpha$ ranges the interval $[0,1]$, with $\alpha=0$ meaning no damage while $\alpha=1$ corresponds to a completely disintegrated rock. To make the damage model relatively simple without causing unnecessary analytic complications (leading e.g. to implementing the concept of nonsimple materials), the simplest modelling assumption that ensures the mentioned constraint $0 \leq \alpha \leq 1$ is

$$
\partial_{\alpha} \mathcal{G}_{\mathrm{E}}(0, e)=0=\partial_{\alpha} \mathcal{G}_{\mathrm{E}}(1, e) .
$$

This facilitates the analysis of the model, sketched in Appendix below. Without going into details here, we can here summarize the main theoretical result as:

Proposition 2.1. The initial-boundary-value problem (2.10)-(2.16) admits a weak solution in the sense of Definition 5.1 below. This solution conserves energy in the sense that (2.15) holds when integrated over any time interval $[0, t]$ with $0<t \leq T$. 


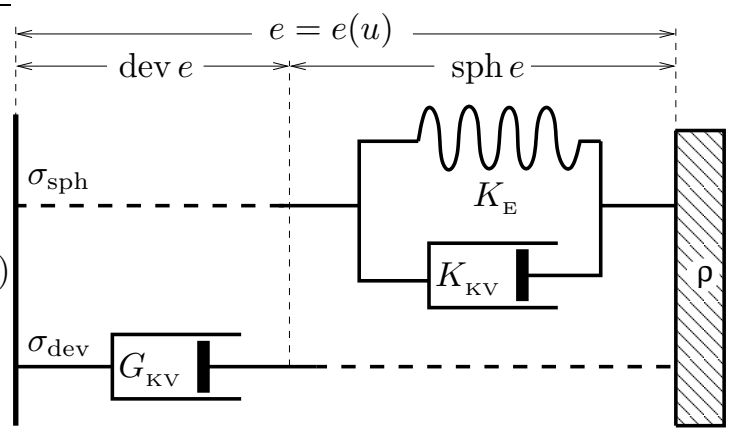

Figure 3: Schematic diagramme for the Boger-fluid rheology, resulting from Fig. 2 when sending $K_{\mathrm{MX}} \rightarrow \infty, G_{\mathrm{Mx}} \rightarrow \infty$, and $G_{\mathrm{E}}(\alpha) \rightarrow 0$. This model is considered in $\Omega_{\mathrm{F}}$ (=the outer core and oceans) coupled with the model from Figure 2 in $\Omega_{\mathrm{S}}$ (=the mantle and the inner core).

Remark 2.2 (Activated creep: plasticity). Some other modification of the "solid" model used in geophysical literature modifies the evolution of the creep $\pi$ to be activated in the spirit what it standardly used in plasticity. Then $\zeta$ should be augmented by a term like $|\operatorname{dev} \pi|$ and, to facilitate mathematical analysis, then $\varphi$ is to be augmented by the term $|\nabla \operatorname{dev} \pi|^{2}$. Then $\pi$ is called an inelastic strain, rather than a Maxwellian creep. This model may be relevant in particular to provide an additional dissipation of energy important during big earthquakes and damage-dependent yield stress that may facilitate fast rupture.

Remark 2.3 (Geopotential). In the standard presentation of the selfgraviting model, the evolving potential $\phi$ is rather a perturbation of another gravitational potential (constant in time), resulted from a steady-state equilibrium configuration at a chosen initial time with the Coriolis force zero and the centrifugal force $\varrho \omega \times(\omega \times x)$. The sum of these two is sometimes referred as a geopotential. Then another force occurs in (2.10a), causing the body $\Omega$ to be pre-stressed. Cf. e.g. [4,6, 32].

\section{Towards Boger viscoelastic fluid in the outer core and oceans}

A standard specification of so-called Boger's viscoelastic fluids is as constant-viscosity elastic (non-Newtonian) fluids that behave as both liquids and solids. Originally, this concept was devised rather for materials like dilute polymer solutions. Yet, such (idealized) material may model the fluid outer core in the Earth, which is a $2200 \mathrm{~km}$ thick layer between the (rather solid) inner core of the radius about $1300 \mathrm{~km}$ and the (rather solid) mantle, cf. Fig. 1. The elasticity in the volumetric part is essential because it allows for a propagation of $\mathrm{P}$-waves, which is a well documented phenomenon in the outer core, while S-waves are practically not penetrating this region because of its fluidic character as far as the shear response concerns, cf. Figure 3. Knowing quite reliably the speed $v_{\mathrm{P}}$ of the $\mathrm{P}$-waves and the mass density $\varrho$ depending on the depth, the elastic bulk modulus $K_{\mathrm{E}}=K_{\mathrm{E}}(x)$ in the outer core is easy to be seen from the formula $v_{\mathrm{P}}=\sqrt{M / \varrho}$ where $M=\lambda+2 G_{\mathrm{E}}=K_{\mathrm{E}}+\frac{4}{3} G_{\mathrm{E}}$ is the so-called P-wave modulus; here $G_{\mathrm{E}}=G_{\mathrm{E}}(\alpha)$ in (2.7). In the elastic fluid $G_{\mathrm{E}}=0$ so that $M=K_{\mathrm{E}}$. One can indeed determine simply as $K_{\mathrm{E}}=\varrho v_{\mathrm{P}}^{2}$ when knowing $\varrho$ and $v_{\mathrm{P}}$. According the reliably documented speed of the seismic P-waves varying from $8 \mathrm{~km} / \mathrm{s}$ (top) to $10 \mathrm{~km} / \mathrm{s}$ (bottom) and the mass density varying from $10000 \mathrm{~kg} / \mathrm{m}^{3}$ (top) to $12000 \mathrm{~kg} / \mathrm{m}^{3}$ (bottom), the elastic bulk modulus ranges from $K_{\mathrm{E}}=640 \mathrm{GPa}$ (top) to $1200 \mathrm{GPa}$ (bottom).

For oceanic layers, it is relevant that the bulk modulus of seawater is about $K_{\mathrm{E}}=M=$ 2.3 $\mathrm{GPa}$ (increasing with pressure) which is, e.g., about $80 \times$ smaller than in steel and about $5 \times$ smaller than in the crust, and thus water is remarkably elastically compressible. Modelling oceans as elastic fluid is thus relevant for propagation seismic P-waves, which may manifest themselves on the surface by the Tsunami waves, cf. e.g. 19, 30. Note that, counting the speed of P-waves close to the sea surface where $\varrho \sim 10^{3} \mathrm{~kg} / \mathrm{m}^{3}$ is $v_{\mathrm{P}}=\sqrt{M / \varrho} \sim 1.5 \mathrm{~km} / \mathrm{s}$, which is the sound speed, so underwater acoustics exploits the same mechanism as seismic P-waves.

In addition to the elastic response, there is a (rather small) viscosity $\left(K_{\mathrm{KV}}, G_{\mathrm{KV}}\right)$ in the fluidic 
outer core and in oceans, too. In the outer core, some data indicates it of the order $10^{-2} \mathrm{Pas}$, cf. [31, while some uncertainty and big variation within depth $10^{4} \mathrm{~Pa}$ s seems also documented in literature, see e.g. [27, 28, The viscosity of the water is even lower, of the order $10^{-3} \mathrm{Pas}$, varying with temperature and pressure.

Here we can come to a viscoelastic (Boger's) fluid as a limit from the previous damageable Jeffreys' rheology when sending $G_{\mathrm{E}}(\alpha) \rightarrow 0$ so that, in particular, the resulting material naturally will not be subjected to any damage, and simultaneously $K_{\mathrm{MX}} \rightarrow \infty$, and $G_{\mathrm{MX}} \rightarrow \infty$. As a result, $\pi \rightarrow 0$ and eventually (2.10b) is to hold only on $\Omega_{\mathrm{S}}$ instead of the whole $\Omega$ while (2.10c) still holds on the whole $\Omega$ but $\partial_{\alpha} \mathcal{G}_{\mathrm{E}}\left(\alpha, e_{\mathrm{el}}\right)$ is zero on $\Omega_{\mathrm{F}}$ and values of the damage $\alpha$ are actually irrelevant 2.10a on $\Omega_{\mathrm{F}}$.

More specifically, the system that results from (2.10) by this limit procedure looks as:

$$
\begin{aligned}
& \varrho \ddot{u}-\operatorname{div}\left(\sigma_{\mathrm{sph}}+\sigma_{\mathrm{dev}}\right)+\varrho(\omega \times(\omega \times(x+u))+2 \omega \times \dot{u}+\nabla \phi)=0 \quad \text { in } \Omega \\
& \text { with } \sigma_{\mathrm{sph}}= \begin{cases}3 K_{\mathrm{KV}} \operatorname{sph} \dot{e}_{\mathrm{el}}+3 K_{\mathrm{E}} \mathrm{sph} e_{\mathrm{el}} & \text { in } \Omega_{\mathrm{S}}, \\
3 K_{\mathrm{KV}} \operatorname{sph} e(\dot{u})+3 K_{\mathrm{E}} \operatorname{sph} e(u) & \text { in } \Omega_{\mathrm{F}},\end{cases} \\
& \text { and } \sigma_{\mathrm{dev}}= \begin{cases}2 G_{\mathrm{KV}} \operatorname{dev} \dot{e}_{\mathrm{el}}+\partial_{e} \mathcal{G}_{\mathrm{E}}\left(\alpha, \operatorname{dev} e_{\mathrm{el}}\right) & \text { in } \Omega_{\mathrm{S}}, \\
2 G_{\mathrm{KV}} \operatorname{sph} e(\dot{u}) & \text { in } \Omega_{\mathrm{F}},\end{cases} \\
& \mathbb{D}_{\mathrm{MX}} \dot{\pi}=\sigma_{\mathrm{sph}}+\sigma_{\mathrm{dev}} \\
& \text { in } \Omega_{\mathrm{S}} \text {, } \\
& \partial \eta(\dot{\alpha})+\partial_{\alpha} G_{\mathrm{E}}\left(\alpha, \operatorname{dev} e_{\mathrm{el}}\right)-\operatorname{div}(\kappa \nabla \alpha) \ni 0 \\
& \text { in } \Omega_{\mathrm{S}} \text {, } \\
& \operatorname{div}\left(\frac{1}{4 \pi g} \nabla \phi+\varrho u\right)=\varrho+\varrho_{\text {ext }}(t) \\
& \text { in } \mathbb{R}^{3} \text {. }
\end{aligned}
$$

The interface conditions for displacement/stress on the interior boundaries between the mantle and the outer core and the inner and the outer core as well between the mantle (crust) and the oceans are automatically involved as (3.1a) holds on the whole $\Omega$. In particular, the stress-vector equilibrium and continuity of the displacement across these interfaces are automatically involved and does not need to be written explicitly. On the other hand, note in particular that both $\pi$ and $\alpha$ in (3.1) are now needed and defined only in the solid part $\Omega_{\mathrm{S}}$. Therefore, the condition for the "flux" of $\alpha$ is now to be prescribed on all interior boundaries (i.e. on the mantle/core and mantle/oceans and inner/outer-core interfaces). More specifically, (2.16c) is to be replaced by

$$
(\kappa \nabla \alpha) \cdot \vec{n}=0 \text { on } \partial \Omega_{\mathrm{S}} .
$$

We can justify this limit-passage scenario rigorously. To this goal, let us choose

$$
\begin{aligned}
& G_{\mathrm{MX}, \varepsilon}(x)=\left\{\begin{array}{l}
G_{\mathrm{MX}}(x) / \varepsilon, \\
G_{\mathrm{MX}}(x),
\end{array} \quad K_{\mathrm{MX}, \varepsilon}(x)= \begin{cases}K_{\mathrm{MX}}(x) / \varepsilon & \text { for } x \in \Omega_{\mathrm{F}}, \\
K_{\mathrm{MX}}(x) & \text { for } x \in \Omega_{\mathrm{S}},\end{cases} \right. \\
& \mathcal{G}_{\mathrm{E}, \varepsilon}(x, \alpha, e)=\left\{\begin{array}{ll}
\varepsilon \mathcal{G}_{\mathrm{E}}(x, \alpha, e), \\
\mathcal{G}_{\mathrm{E}}(x, \alpha, e),
\end{array} \quad \kappa_{\varepsilon}(x)=\left\{\begin{array}{cc}
\varepsilon \kappa(x) & \text { for } x \in \Omega_{\mathrm{F}}, \\
\kappa(x) & \text { for } x \in \Omega_{\mathrm{S}},
\end{array}\right.\right. \\
& \eta_{\varepsilon}(x, \dot{\alpha})= \begin{cases}\varepsilon \eta(x, \dot{\alpha}) & \text { for } x \in \Omega_{\mathrm{F}}, \\
\eta(x, \dot{\alpha}) & \text { for } x \in \Omega_{\mathrm{S}},\end{cases}
\end{aligned}
$$

while the other visco-elastic moduli $K_{\mathrm{E}}, K_{\mathrm{KV}}$, and $G_{\mathrm{KV}}$ are kept fixed. The following statement will be made more specific and proved in the Appendix:

Proposition 3.1. Let be some solution $\left(u_{\varepsilon}, \pi_{\varepsilon}, \alpha_{\varepsilon}, \phi_{\varepsilon}\right)$ of the initial-boundary-value problem (2.10)-(2.16) with the data (3.3), which do exist due to Proposition 2.1. Then $\left(u_{\varepsilon},\left.\pi_{\varepsilon}\right|_{Q_{\mathrm{S}}},\left.\alpha_{\varepsilon}\right|_{Q_{\mathrm{S}}}, \phi_{\varepsilon}\right)$ converge (in terms of subsequences) for $\varepsilon \rightarrow 0$ to weak solutions to (3.1) with the initial/boundary conditions (2.16a,b)-(3.2). In particular, a weak solution to the initialboundary-value problem (3.1)-(2.16a,b)-(3.2) in the sense of Definition 5.2 below does exist. In addition, this solution conserves energy. Moreover, the energy dissipated through the Maxwelltype attenuation and by damage in the fluidic regions $\Omega_{\mathrm{F}}$ over the time interval I converges to 
zero, i.e.

$$
\int_{Q_{\mathrm{F}}} \frac{3}{2} K_{\mathrm{MX}, \varepsilon}\left|\operatorname{sph} \dot{\pi}_{\varepsilon}\right|^{2}+G_{\mathrm{MX}, \varepsilon}\left|\operatorname{dev} \dot{\pi}_{\varepsilon}\right|^{2}+\dot{\alpha}_{\varepsilon} \partial \eta\left(\dot{\alpha}_{\varepsilon}\right) \mathrm{d} x \mathrm{~d} t \rightarrow 0 \quad \text { for } \varepsilon \rightarrow 0 .
$$

It should be emphasized that, although (3.4) similarly as (4.3) below is intuitively quite clear and generally expected in geophysical literature,its rigorous proof is not entirely trivial and relies on a regularity which is rather automatic in linear systems but may be highly nontrivial or even false in nonlinear hyperbolic systems, as basically also here, cf. [21,25].

Remark 3.2. Instead of $G_{\mathrm{Mx}} \rightarrow \infty$ and $G_{\mathrm{E}}(\alpha) \rightarrow 0$, one can think about sending $G_{\mathrm{KV}} \rightarrow \infty$. Then, assuming the initial condition $\left.\operatorname{dev} e_{\mathrm{el}}\right|_{t=0}=0$, we would have $\operatorname{dev} e_{\mathrm{el}} \rightarrow 0$. Hence, in the limit we would see the model from Fig. 3 only with $G_{\mathrm{KV}}$ replaced by $G_{\mathrm{Mx}}$. Since the viscosity in fluidic regions is typically small (as discuss in particular in the next section 4) like also $G_{\mathrm{KV}}$ while $G_{\mathrm{Mx}}$ is typically large in solid-like geophysical materials, our choice (3.3) is more straightforward. Another difference would be that, instead of $\pi \rightarrow 0$, we would have only $\operatorname{sph} \pi \rightarrow 0$ while $\operatorname{dev} \pi \rightarrow \operatorname{dev} e(u)$.

Remark 3.3. Actually, the limit $K_{\mathrm{Mx}} \rightarrow \infty$ is relevant also in the solid part $\Omega_{\mathrm{S}}$. This causes $\operatorname{sph} \pi \rightarrow 0$ and in the limit one obtains a model where $\pi$ is trace-free and accumulates only shear strain, which is the most common ansatz in plasticity and creep, and in geophysical modelling, too.

Remark 3.4 (Incompressible Stokes-fluid limit). Passing simultaneously $K_{\mathrm{E}} \rightarrow \infty$, we obtain in the limit the Stokes incompressible fluid. The bulk viscosity $K_{\mathrm{KV}}$ then becomes irrelevant, so that only the shear viscosity $G_{\mathrm{KV}}$ remains relevant in Figure 3. Such model is a great idealization and has limited application because, beside $v_{\mathrm{S}} \rightarrow 0$, now nonphysically $v_{\mathrm{P}} \rightarrow \infty$. In particular, in this limit, $\operatorname{sph} e(u)$ is constant in time, namely $\operatorname{sph} e(u(x, t))=\frac{1}{3} \operatorname{div} u_{0}(x) \mathbb{I}$. Therefore $\operatorname{div} \dot{u}=0$, which expresses the usual incompressibility condition. Such models are often used in geophysics in short-time-scale models when the convective term $\varrho(\dot{u} \cdot \nabla) \dot{u}$, occurring in Navier-Stokes equation, can be neglected. Cf. e.g. [29] for self-gravitating incompressible Stokes model in layered geoid.

\section{Suppressing viscosity towards purely elastic fluid}

As already said, the viscosity $\left(K_{\mathrm{KV}}, G_{\mathrm{KV}}\right)$ in the fluidic domains is only very small (and even not certainly known in deep parts of the outer core). Note also that, when $G_{\mathrm{E}}=0$ in the fluidic domains, $G_{\mathrm{KV}}$ is in the position of the Maxwellian viscosity. For example, in mantle, $10^{2}-10^{4} \mathrm{Pas}$ is considered in [17]. As for the fluidic regions, as said above, the oceans exhibit viscosity around $10^{-3} \mathrm{Pas}$ while the outer core around $10^{-2}-10^{4} \mathrm{Pas}$. In any case, these viscosities are much smaller than typical viscosity in the crust which is of the order $10^{22}-10^{24} \mathrm{Pas}$ or in the inner core of the order about $10^{14}-10^{15} \mathrm{Pas}$, see e.g. [13]. This certainly gives a good motivation to study the asymptotics when this viscosity goes to zero. In the limit, it yields an inviscid, purely elastic model where the Hooke elasticity counts only with the spherical response while the shear-stress-free response imitates the ideal inviscid fluid; sometimes, such sort of models are called compressible Euler fluids. Such materials are called elastic fluids, or more specifically just compressible inviscid fluids. These fluids allow still for propagation of $P$-waves (whose speed is $\varrho^{-1 / 2} K_{\mathrm{E}}^{1 / 2}$ as already mentioned) while S-waves are completely excluded.

The resulted system is again (3.17, d-f) but now with

$$
\begin{aligned}
\sigma_{\mathrm{sph}} & = \begin{cases}3 K_{\mathrm{KV}} \operatorname{sph}(e(\dot{u})-\dot{\pi})+3 K_{\mathrm{E}} \operatorname{sph}(e(u)-\pi) & \text { in } \Omega_{\mathrm{S}}, \\
3 K_{\mathrm{E}} \operatorname{sph} e(u) & \text { in } \Omega_{\mathrm{F}},\end{cases} \\
\sigma_{\mathrm{dev}} & = \begin{cases}2 G_{\mathrm{KV}} \operatorname{dev}(e(\dot{u})-\dot{\pi})+\partial_{e} \mathcal{G}_{\mathrm{E}}(\alpha, \operatorname{dev}(e(u)-\pi)) & \text { in } \Omega_{\mathrm{S}} \\
0 & \text { in } \Omega_{\mathrm{F}}\end{cases}
\end{aligned}
$$

The weak formulation for the limit problem as far as the force equilibrium (3.1 $\mathrm{a}, \mathrm{d})$ with (4.1) concerns arises when testing (3.1a) by a smooth test function $v$ with $v(T)=0$ and $\operatorname{dev} e(v)=0$ on 
$Q_{\mathrm{F}}$ and making by part integration in time and applying Green formula in space, cf. Definition 5.5 in the Appendix.

We can justify this limit-passage scenario rigorously. To this goal, let us choose

$$
K_{\mathrm{KV}, \varepsilon}(x)=\left\{\begin{array}{c}
\varepsilon K_{\mathrm{KV}}(x), \quad G_{\mathrm{KV}, \varepsilon}(x)=\left\{\begin{array}{c}
\varepsilon G_{\mathrm{KV}}(x) \text { for } x \in \Omega_{\mathrm{F}}, \\
K_{\mathrm{KV}}(x),
\end{array} G_{\mathrm{KV}}(x) \text { for } x \in \Omega_{\mathrm{S}},\right.
\end{array}\right.
$$

while $G_{\mathrm{E}}(\alpha)=0$ and $K_{\mathrm{Mx}}=G_{\mathrm{Mx}}=\infty$ on $\Omega_{\mathrm{F}}$, resulting already from the limit in Section 3. The following statement will be proved in the Appendix:

Proposition 4.1. If the initial conditions are enough smooth (cf. the assumption in Definition 5.5 below), the solutions $\left(u_{\varepsilon}, \pi_{\varepsilon}, \alpha_{\varepsilon}, \phi_{\varepsilon}\right)$ of the initial-boundary-value problem (3.1)(2.16a,b)-(3.2) with the data (4.2), which does exist due to Proposition 3.1, converge (in terms of subsequences) for $\varepsilon \rightarrow 0$ to weak solutions to (3.1 $\mathrm{a}, \mathrm{d}-\mathrm{f})-(2.16 \mathrm{~b}, \mathrm{~b})-(\mathrm{3.2})$ with (4.1) . In particular, a weak solution to this initial-boundary-value problem in the sense of Definition 5.5 below does exist. Moreover, this solution conserves energy and the energy dissipated through the Kelvin-Voigt-type attenuation in the fluidic regions $\Omega_{\mathrm{F}}$ over the time interval I converges to zero, i.e.

$$
\varepsilon \int_{Q_{\mathrm{F}}} \frac{3}{2} K_{\mathrm{KV}}\left|\operatorname{sph} e\left(\dot{u}_{\varepsilon}\right)\right|^{2}+G_{\mathrm{KV}}\left|\operatorname{dev} e\left(\dot{u}_{\varepsilon}\right)\right|^{2} \mathrm{~d} x \mathrm{~d} t \rightarrow 0 \quad \text { for } \varepsilon \rightarrow 0 .
$$

\section{Appendix: analysis sketched}

In what follows, we will use the (standard) notation for the Lebesgue $L^{p}$-spaces and $W^{k, p}$ for Sobolev spaces whose $k$-th distributional derivatives are in $L^{p}$-spaces, and the abbreviation $H^{k}=W^{k, 2}$. Moreover, we will use the standard notation $p^{\prime}=p /(p-1)$. In the vectorial case, we will write $L^{p}\left(\Omega ; \mathbb{R}^{3}\right) \cong L^{p}(\Omega)^{3}$ and $W^{1, p}\left(\Omega ; \mathbb{R}^{3}\right) \cong W^{1, p}(\Omega)^{3}$. For the fixed time interval $I=[0, T]$, we denote by $L^{p}(I ; X)$ the standard Bochner space of Bochner-measurable mappings $I \rightarrow X$ with $X$ a Banach space. Also, $W^{k, p}(I ; X)$ denotes the Banach space of mappings from $L^{p}(I ; X)$ whose $k$-th distributional derivative in time is also in $L^{p}(I ; X)$. The dual space to $X$ will be denoted by $X^{*}$. The scalar product between vectors, matrices, or 3rd-order tensors will be denoted by ".", ":", or " : ", respectively. Finally, in what follows, $K$ denotes a positive, possibly large constant.

We will impose the basic assumptions on the data and incorporate them directly into Definitions 5.15.5. In particular,

$$
\begin{aligned}
& \inf _{x \in \Omega} \varrho(x)>0, \quad \varrho=0 \text { on } \mathbb{R}^{3} \backslash \Omega, \quad \varrho \in L^{\infty}(\Omega) \cap W^{1,3}(\Omega), \\
& \varrho_{\text {ext }} \in W^{1,1}\left(I ; L^{\infty}\left(\mathbb{R}^{3}\right)\right), \quad \varrho_{\text {ext }}=0 \text { outside a bounded set in } I \times \mathbb{R}^{3}, \\
& K_{\mathrm{E}}, G_{\mathrm{KV}}, K_{\mathrm{KV}}, G_{\mathrm{MX}}, K_{\mathrm{MX}}, \mathcal{G}_{\mathrm{E}}(\cdot, \alpha, e), \kappa: \Omega \rightarrow[0, \infty) \text { measurable and } \\
& \quad \inf _{x \in \Omega} \min \left(G_{\mathrm{KV}}(x), K_{\mathrm{KV}}(x), G_{\mathrm{MX}}(x), K_{\mathrm{MX}}(x), K_{\mathrm{E}}(x), \kappa\right)>0, \\
& \quad \sup _{x \in \Omega_{\mathrm{S}} \max }\left(K_{\mathrm{KV}}(x) / K_{\mathrm{MX}}(x), G_{\mathrm{KV}}(x) / G_{\mathrm{MX}}(x)\right)<1, \\
& \mathcal{G}_{\mathrm{E}}(x, \cdot, \cdot):[0,1] \times \mathbb{R}^{3 \times 3} \rightarrow[0, \infty) \text { twice continuously differentiable, } \\
& \quad \mathcal{G}_{\mathrm{E}}(x, \alpha, \cdot): \mathbb{R}^{3 \times 3} \rightarrow[0, \infty) \quad \text { convex, } \\
& \quad\left|\partial_{e} \mathcal{G}_{\mathrm{E}}(x, \alpha, e)\right| \leq C(1+|e|), \quad\left|\partial_{\alpha} \mathcal{G}_{\mathrm{E}}(x, \alpha, e)\right| \leq C(1+|e|), \\
& \left|\partial_{e e}^{2} \mathcal{G}_{\mathrm{E}}(x, \alpha, e)\right| \leq C, \quad\left|\partial_{\alpha e}^{2} \mathcal{G}_{\mathrm{E}}(x, \alpha, e)\right| \leq C, \\
& \eta \mathbb{R} \rightarrow \mathbb{R} \quad \text { uniformly convex, } \eta(0)=0, \\
& u_{0} \in H^{1}\left(\Omega ; \mathbb{R}^{3}\right), \quad v_{0} \in L^{2}\left(\Omega ; \mathbb{R}^{3}\right), \quad \pi_{0} \in L^{2}\left(\Omega ; \mathbb{R}^{3 \times 3}\right), \quad \pi_{0}=0 \text { on } \Omega_{\mathrm{F}}, \\
& \text { and } \alpha_{0} \in H^{1}(\Omega) \quad \text { with } 0 \leq \alpha_{0}(x) \leq 1 \text { for all } x \in \Omega .
\end{aligned}
$$

Let us emphasize that (5.1c) is used only for the second limit passage for estimation (5.19) $-(5.20)$ and is well satisfied in geophysical models where the ratio of the Kelvin-Voigt and Maxwell viscosities in solid regions is surely below $10^{-8}$, as said in Section 4, Also, let us emphasize that 
the growth restrictions imposed on $\partial_{\alpha} \mathcal{G}_{\mathrm{E}}$ and $\partial_{\alpha e}^{2} \mathcal{G}_{\mathrm{E}}$ in (5.1d) are compatible with the ansatz (2.7) and used in (5.3b) and for (5.19) below.

We further integrate (2.11) over $Q$ and apply the Green theorem and the by-part integration in time to the term $(v-\dot{\alpha}) \operatorname{div}(\kappa \nabla \alpha)$, cf. Remark 5.4.

Definition 5.1 (Weak solution to (2.10)-(2.16) $)$. The quadruple $(u, \pi, \alpha, \phi)$ is called a weak solution to the initial-boundary-value problem (2.10)-(2.16) provided the data satisfies (5.1) and

$$
\begin{aligned}
& u \in W^{1, \infty}\left(I ; L^{2}\left(\Omega ; \mathbb{R}^{3}\right)\right) \cap H^{1}\left(I ; H^{1}\left(\Omega ; \mathbb{R}^{3}\right)\right), \\
& \pi \in H^{1}\left(I ; L^{2}\left(\Omega ; \mathbb{R}^{3 \times 3}\right)\right), \\
& \alpha \in L^{\infty}\left(I ; H^{1}(\Omega)\right) \cap H^{1}\left(I ; L^{2}(\Omega)\right), \\
& \phi \in L^{\infty}\left(I ; H^{1}\left(\mathbb{R}^{3}\right)\right),
\end{aligned}
$$

and the integral identity

$$
\int_{Q} \sigma: e(v)-\varrho \dot{u} \cdot \dot{v}+f \cdot v \mathrm{~d} x \mathrm{~d} t=\int_{\Omega} \varrho v_{0} \cdot v(0) \mathrm{d} x
$$

holds for any $v \in H^{1}\left(Q ; \mathbb{R}^{3}\right)$ with $v(T)=0$ and with $\sigma$ and $f$ from (2.10a),

$$
\begin{aligned}
& \int_{Q}(v-\dot{\alpha}) \partial_{\alpha} \mathcal{G}_{\mathrm{E}}(\alpha, \operatorname{dev}(e(u)-\pi))+\kappa \nabla \alpha \cdot \nabla v+\eta(v) \mathrm{d} x \mathrm{~d} t \\
&+\int_{\Omega} \frac{\kappa}{2}\left|\nabla \alpha_{0}\right|^{2} \mathrm{~d} x \geq \int_{Q} \eta(\dot{\alpha}) \mathrm{d} x \mathrm{~d} t+\int_{\Omega} \frac{\kappa}{2}|\nabla \alpha(T)|^{2} \mathrm{~d} x
\end{aligned}
$$

for all $v \in L^{2}\left(I ; H^{1}(\Omega)\right)$,

$$
\int_{I \times \mathbb{R}^{3}}\left(\frac{1}{4 \pi g} \nabla \phi+\varrho u\right) \cdot \nabla v+\left(\varrho+\varrho_{\mathrm{ext}}\right) v \mathrm{~d} x \mathrm{~d} t=0
$$

for all $v \in L^{2}\left(I ; H^{1}\left(\mathbb{R}^{3}\right)\right)$, and also $(2.10 \mathrm{~b})$ holds a.e. on $Q$, and eventually the resting initial conditions $u(0)=u_{0}, \pi(0)=\pi_{0}$, and $\alpha(0)=\alpha_{0}$ hold a.e. on $\Omega$.

Sketched proof of Proposition 2.1. Without going into details, we may expect that we applied some approximation method (e.g. a Galerkin-type approximation in space) to obtain some approximate solution to the initial-boundary-value problem (2.10)-(2.16) which, in principle, can be implemented on computers e.g. by the finite-element method. Then the a-priori estimates in the space as in (5.2) hold also for the approximate solutions, which may be interpreted as a numerical stability of the specific approximation scheme. This approximation leads (after smoothening of the potential $\eta$ ) to the initial-value problem for a system of ordinary-differential equations. Existence of its solution, let us denote it by $\left(u_{k}, \pi_{k}, \alpha_{k}, \phi_{k}\right)$ with $k \in \mathbb{N}$ referring to the finite-dimensional subspaces used for the Galerkin discretisation, can be proved by successivecontinuation argument, using the $L^{\infty}(I)$-estimates below. Here we may assume that the initial conditions lie in the finite-dimensional spaces used for the Galerkin approximation so that no further approximation is needed.

The energy balance (2.15) can serve to see basic apriori estimates and to perform analysis of the system (2.10). Integrating (2.15) over $[0, t]$ and using the by-part integration in time for the power of tidal load $\varrho_{\text {ext }} \dot{\phi}$ and the notation $(2.9 \mathrm{~b}, \mathrm{c})$, we write

$$
\begin{aligned}
\int_{\Omega}\left(\frac{\varrho}{2}\left|\dot{u}_{k}(t)\right|^{2}+\frac{3}{2} K_{\mathrm{E}}\left|\operatorname{sph} e_{\mathrm{el}, k}(t)\right|^{2}+\mathcal{G}_{\mathrm{E}}\left(\alpha_{k}(t), \operatorname{dev} e_{\mathrm{el}, k}(t)\right)\right. \\
\left.+\frac{\kappa}{2}\left|\nabla \alpha_{k}(t)\right|^{2}+\frac{1}{2} \varrho\left|\omega \cdot\left(x+u_{k}(t)\right)\right|^{2}\right) \mathrm{d} x+\int_{\mathbb{R}^{3}} \frac{1}{8 \pi g}\left|\nabla \phi_{k}(t)\right|^{2} \mathrm{~d} x \\
+\int_{0}^{t} \int_{\Omega} \mathbb{D}_{\mathrm{KV}} \dot{e}_{\mathrm{el}, k}: \dot{e}_{\mathrm{el}, k}+\mathbb{D}_{\mathrm{Mx}} \dot{\pi}_{k}: \dot{\pi}_{k}+\dot{\alpha}_{k} \eta^{\prime}\left(\dot{\alpha}_{k}\right) \mathrm{d} x \mathrm{~d} t \\
\leq \int_{\Omega}-\varrho \nabla \phi_{k}(t) \cdot u_{k}(t)-\varrho \phi_{k}(t) \mathrm{d} x \\
\quad+\int_{\mathbb{R}^{3}} \varrho_{\mathrm{ext}}(t) \phi_{k}(t) \mathrm{d} x-\int_{0}^{t} \int_{\mathbb{R}^{3}} \dot{\varrho}_{\mathrm{ext}} \phi_{k} \mathrm{~d} x \mathrm{~d} t+E_{0}
\end{aligned}
$$


with $e_{\mathrm{el}, k}=e\left(u_{k}\right)-\pi_{k}$ and with the upper bound for the initial energy

$$
\begin{aligned}
E_{0}=\int_{\Omega} \frac{\varrho}{2}\left|v_{0}\right|^{2}+\frac{3}{2} K_{\mathrm{E}}\left|\mathrm{sph} e_{\mathrm{el}, 0}\right|^{2}+\mathcal{G}_{\mathrm{E}}\left(\alpha_{0}, e_{\mathrm{el}, 0}\right)+\varrho \phi(0)+\varrho \nabla \phi(0) \cdot u_{0} \\
+\frac{1}{2} \varrho\left|\omega \cdot\left(x+u_{0}\right)\right|^{2}+\frac{\kappa}{2}\left|\nabla \alpha_{0}\right|^{2} \mathrm{~d} x+\int_{\mathbb{R}^{3}} \varrho_{\mathrm{ext}}(0, x) \phi(0, x) \mathrm{d} x,
\end{aligned}
$$

where $e_{\mathrm{el}, 0}=e\left(u_{0}\right)-\pi_{0}$ and where $\phi(0, x) \in H^{1}(\Omega)$ is the gravitational potential solving (2.10d) for $t=0$. The inequality in (5.4) have arisen from the energy equality (2.15) written for the approximate solution and integrated over the time interval $[0, t]$ by forgetting some parts of the centrifugal potential with a guaranteed sign. The non-coercive contribution of the centrifugal force on the right-hand side of (5.4) is to be estimated by Hölder's inequality as

$$
\begin{aligned}
\int_{\Omega} \frac{1}{2} \varrho|\omega|^{2}\left|x+u_{k}(t)\right|^{2} \mathrm{~d} x & =\int_{\Omega} \frac{1}{2} \varrho|\omega|^{2}\left|x+u_{0}(x)+\int_{0}^{t} \dot{u}_{k}(\tau, x) \mathrm{d} \tau\right|^{2} \mathrm{~d} x \\
& \leq C\left(1+\int_{0}^{t}\left\|\dot{u}_{k}(\tau)\right\|_{L^{2}\left(\Omega ; \mathbb{R}^{3}\right)}^{2} \mathrm{~d} \tau\right)
\end{aligned}
$$

with some constant $C$, and then treated by Gronwall's inequality, exploiting the kinetic energy on the left-hand side of (5.4). Furthermore, we can estimate $\int_{\Omega} \nabla \phi_{k} \cdot u_{k} \mathrm{~d} x \leq \epsilon\left\|\nabla \phi_{k}\right\|_{L^{2}\left(\Omega ; \mathbb{R}^{3}\right)}^{2}+$ $C_{\epsilon}\left(\int_{0}^{t}\left\|\dot{u}_{k}\right\|_{L^{2}\left(\Omega ; \mathbb{R}^{3}\right)}^{2} \mathrm{~d} t+\left\|u_{0}\right\|_{L^{2}\left(\Omega ; \mathbb{R}^{3}\right)}^{2}\right)$. Also use the estimate $\left\|\phi_{k}\right\|_{L^{2}(\Omega)} \leq C\left\|\nabla \phi_{k}\right\|_{L^{2}\left(\mathbb{R}^{3} ; \mathbb{R}^{3}\right)}$ relying on the boundedness of $\Omega$, provided $\phi(\infty)=0$ which is a standard "boundary" condition for the gravitational potential used in geophysics. The last integral on the right-hand side of (5.4) bears the estimation

$$
\begin{aligned}
& \int_{0}^{t} \int_{\mathbb{R}^{3}} \dot{\varrho}_{\text {ext }} \phi_{k} \mathrm{~d} x \mathrm{~d} t \leq \int_{0}^{t}\left\|\dot{\varrho}_{\text {ext }}\right\|_{L^{\infty}\left(\mathbb{R}^{3}\right)}\left\|\phi_{k}\right\|_{L^{1}\left(\mathbb{R}^{3}\right)} \mathrm{d} t \\
& \leq C \int_{0}^{t}\left\|\dot{\varrho}_{\text {ext }}\right\|_{L^{\infty}\left(\mathbb{R}^{3}\right)}\left\|\nabla \phi_{k}\right\|_{L^{2}\left(\mathbb{R}^{3} ; \mathbb{R}^{3}\right)} \mathrm{d} t \leq \int_{0}^{t}\left\|\dot{\varrho}_{\text {ext }}\right\|_{L^{\infty}\left(\mathbb{R}^{3}\right)}\left(1+\left\|\phi_{k}\right\|_{H^{1}\left(\mathbb{R}^{3}\right)}^{2}\right) \mathrm{d} t,
\end{aligned}
$$

where we used $\left\|\phi_{k}\right\|_{L^{1}\left(\mathbb{R}^{3}\right)} \leq C\left\|\nabla \phi_{k}\right\|_{L^{2}\left(\mathbb{R}^{3} ; \mathbb{R}^{3}\right)}$; here the "boundary" condition $\phi_{k}(\infty)=0$ together with $\varrho+\varrho_{\text {ext }}$ compactly supported is used. Altogether, using the Gronwall inequality, we obtain the a-priori estimates for the approximate solution:

$$
\begin{aligned}
& \left\|u_{k}\right\|_{W^{1, \infty}\left(I ; L^{2}\left(\Omega ; \mathbb{R}^{3}\right)\right) \cap H^{1}\left(I ; H^{1}\left(\Omega ; \mathbb{R}^{3}\right)\right)} \leq C, \\
& \left\|\pi_{k}\right\|_{H^{1}\left(I ; L^{2}\left(\Omega ; \mathbb{R}^{3 \times 3}\right)\right)} \leq C, \\
& \left\|\alpha_{k}\right\|_{L^{\infty}\left(I ; H^{1}(\Omega)\right) \cap H^{1}\left(I ; L^{2}(\Omega)\right)} \leq C, \\
& \left\|\phi_{k}\right\|_{L^{\infty}\left(I ; H^{1}\left(\mathbb{R}^{3}\right)\right)} \leq C .
\end{aligned}
$$

By Banach's selection principle, we consider a weakly* convergent subsequence respecting the topologies specified in (5.7). For the limit passage in the nonlinear term $\partial_{\alpha} \mathcal{G}_{\mathrm{E}}$ in (5.3b), we need to improve it for the strong convergence of $e_{\mathrm{el}, k} \rightarrow e_{\mathrm{el}}$ in $L^{2}\left(Q ; \mathbb{R}^{3 \times 3}\right)$. To this goal, we use the test function $u_{k}-u$ and $\pi_{k}-\pi$ for the Galerkin approximation of (2.10a) and (2.10b), respectively, integrate it over the time interval $[0, t]$, and estimate

$$
\begin{aligned}
& \int_{\Omega} \frac{1}{2} \mathbb{D}_{\mathrm{KV}}\left(e_{\mathrm{el}, k}(t)-e_{\mathrm{el}}(t)\right):\left(e_{\mathrm{el}, k}(t)-e_{\mathrm{el}}(t)\right) \mathrm{d} x \\
& \leq \frac{1}{2} \int_{\Omega} \mathbb{D}_{\mathrm{KV}}\left(e_{\mathrm{el}, k}(t)-e_{\mathrm{el}}(t)\right):\left(e_{\mathrm{el}, k}(t)-e_{\mathrm{el}}(t)\right)+\mathbb{D}_{\mathrm{MX}}\left(\pi_{k}(t)-\pi(t)\right):\left(\pi_{k}(t)-\pi(t)\right) \mathrm{d} x \\
& +\int_{0}^{t} \int_{\Omega} K_{\mathrm{E}}\left|\operatorname{sph}\left(e_{\mathrm{el}, k}-e_{\mathrm{el}}\right)\right|^{2}+\left(\partial_{e} \mathcal{G}_{\mathrm{E}}\left(\alpha_{k}, e_{\mathrm{el}, k}\right)-\partial_{e} \mathcal{G}_{\mathrm{E}}\left(\alpha_{k}, e_{\mathrm{el}}\right)\right):\left(e_{\mathrm{el}, k}-e_{\mathrm{el}}\right) \mathrm{d} x \mathrm{~d} t \\
& =\int_{0}^{t} \int_{\Omega}-\left(\varrho \ddot{u}_{k}+f_{k}\right) \cdot\left(u_{k}-u\right) \\
& \quad-\left(\mathbb{D}_{\mathrm{KV}} \dot{e}_{\mathrm{el}}+K_{\mathrm{E}} \mathrm{sph} e_{\mathrm{el}}+\partial_{e} \mathcal{G}_{\mathrm{E}}\left(\alpha_{k}, e_{\mathrm{el}}\right)\right):\left(e_{\mathrm{el}, k}-e_{\mathrm{el}}\right)-\mathbb{D}_{\mathrm{MX}} \dot{\pi}:\left(\pi_{k}-\pi\right) \mathrm{d} x \mathrm{~d} t
\end{aligned}
$$




$$
\begin{gathered}
=\int_{0}^{t} \int_{\Omega} \varrho \dot{u}_{k} \cdot\left(\dot{u}_{k}-\dot{u}\right)-f_{k} \cdot\left(u_{k}-u\right)+\left(\partial_{e} \mathcal{G}_{\mathrm{E}}\left(\alpha, e_{\mathrm{el}}\right)-\partial_{e} \mathcal{G}_{\mathrm{E}}\left(\alpha_{k}, e_{\mathrm{el}}\right)\right):\left(e_{\mathrm{el}, k}-e_{\mathrm{el}}\right) \\
-\left(\mathbb{D}_{\mathrm{KV}} \dot{e}_{\mathrm{el}}+K_{\mathrm{E}} \mathrm{sph} e_{\mathrm{el}}+\partial_{e} \mathcal{G}_{\mathrm{E}}\left(\alpha, e_{\mathrm{el}}\right)\right):\left(e_{\mathrm{el}, k}-e_{\mathrm{el}}\right)-\mathbb{D}_{\mathrm{MX}} \dot{\pi}:\left(\pi_{k}-\pi\right) \mathrm{d} x \mathrm{~d} t \\
-\int_{\Omega} \varrho \dot{u}_{k}(t) \cdot\left(u_{k}(t)-u(t)\right) \mathrm{d} x \rightarrow 0
\end{gathered}
$$

with $f_{k}=\varrho\left(\omega \times\left(\omega \times\left(x+u_{k}\right)\right)+2 \omega \times \dot{u}_{k}+\nabla \phi_{k}\right)$; note that $f_{k}$ is bounded in $L^{2}\left(Q ; \mathbb{R}^{3}\right)$ due to the a-priori estimates $(5.7 \mathrm{~h}, \mathrm{~d})$. Actually, (5.8) is to be understood rather as a conceptual strategy: the mentioned test functions $u_{k}-u$ and $\pi_{k}-\pi$ are not legitimate for the Galerkin approximation and $(u, \pi)$ is still to be approximated strongly to be valued in the respective finite-dimensional subspaces - we omitted these standard technical details for simplicity. For the convergence to 0 in (5.8), we used that $\dot{u}_{k} \rightarrow \dot{u}$ strongly $L^{2}\left(Q ; \mathbb{R}^{3}\right)$ due to the Aubin-Lions theorem, relying on the estimate (5.7a) together with an information about $\ddot{u}$ from the equation (2.10a) itself, and also $\dot{u}_{k}(t)$ is bounded in $L^{2}\left(\Omega ; \mathbb{R}^{3}\right)$ while $u_{k}(t) \rightarrow u(t)$ strongly in $L^{2}\left(\Omega ; \mathbb{R}^{3}\right)$ due to the Rellich compact embedding $H^{1}(\Omega) \subset L^{2}(\Omega)$, and also $\partial_{e} \mathcal{G}_{\mathrm{E}}\left(\alpha_{k}, e_{\mathrm{el}}\right) \rightarrow \partial_{e} \mathcal{G}_{\mathrm{E}}\left(\alpha, e_{\mathrm{el}}\right)$ strongly in $L^{2}\left(Q ; \mathbb{R}_{\mathrm{dev}}^{3 \times 3}\right)$ due to the continuity of the Nemytskiı mapping induced by $\partial_{e} \mathcal{G}_{\mathrm{E}}\left(\cdot, e_{\mathrm{el}}\right)$ and $\alpha_{k} \rightarrow \alpha$ in $L^{2}(Q)$ again just by the Rellich theorem since both $\dot{\alpha}_{k}$ and $\nabla \alpha_{k}$ is estimated in $L^{2}$-spaces. Thus, from (5.8), we obtain $e_{\mathrm{el}, k}(t) \rightarrow e_{\mathrm{el}}(t)$ strongly in $L^{2}\left(\Omega ; \mathbb{R}^{3 \times 3}\right)$ for all $t \in I$. Using it for a general $t \in I$, we obtain $e_{\mathrm{el}, k} \rightarrow e_{\mathrm{el}}$ strongly in $L^{2}\left(Q ; \mathbb{R}^{3 \times 3}\right)$ by the Lebesgue theorem.

The limit passage in the Galerkin approximation towards the integral identities (5.3) is then simple by weak/strong continuity or, in case of the variational inequality (5.3b), also semicontinuity.

For the energy conservation, the essential needed facts are that $\sqrt{\varrho} \ddot{u} \in L^{2}\left(I ; H^{1}\left(\Omega ; \mathbb{R}^{3}\right)^{*}\right)$ is in duality with $\sqrt{\varrho} \dot{u} \in L^{2}\left(I ; H^{1}\left(\Omega ; \mathbb{R}^{3}\right)\right)$ and also that $\operatorname{div}(\kappa \nabla \alpha) \in L^{2}(Q)$ is in duality with $\dot{\alpha} \in L^{2}(Q)$ so that the by-part integration formulas rigorously hold:

$$
\begin{aligned}
& \int_{0}^{t}\langle\sqrt{\varrho} \ddot{u}, \sqrt{\varrho} \dot{u}\rangle \mathrm{d} t=\int_{\Omega} \frac{\varrho}{2}|\dot{u}(t)|^{2}-\frac{\varrho}{2}|\dot{u}(0)|^{2} \mathrm{~d} x, \quad \text { and } \\
& \int_{0}^{t} \int_{\Omega} \operatorname{div}(\kappa \nabla \alpha) \dot{\alpha} \mathrm{d} x \mathrm{~d} t=\int_{\Omega} \frac{\kappa}{2}|\nabla \alpha(0)|^{2}-\frac{\kappa}{2}|\nabla \alpha(t)|^{2} \mathrm{~d} x
\end{aligned}
$$

see e.g. [22, Formulas (7.22) and (12.133b)]. More in detail, for (5.9a) we have used the comparison $\sqrt{\varrho} \ddot{u}=(\operatorname{div} \sigma-f) / \sqrt{\varrho}$ and the estimate

$$
\begin{aligned}
& \|\sqrt{\varrho} \ddot{u}\|_{L^{2}\left(I ; H^{1}\left(\Omega ; \mathbb{R}^{3}\right)^{*}\right)}=\sup _{\|v\|_{L^{2}\left(I ; H^{1}\left(\Omega ; \mathbb{R}^{3}\right)\right)} \leq 1} \int_{Q} \frac{\operatorname{div} \sigma-f}{\sqrt{\varrho}} v \mathrm{~d} x \mathrm{~d} t
\end{aligned}
$$

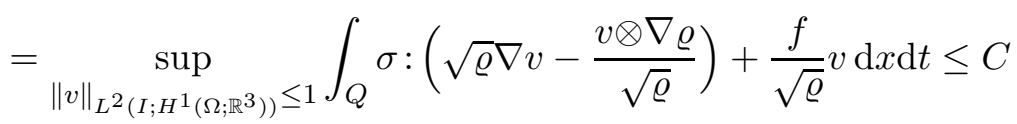

for which the smoothness $\varrho \in W^{1,3}(\Omega)$ is needed due to the occurrence of $\nabla \varrho$ in (5.10), while for (5.9b) we have used the comparison $\operatorname{div}(\kappa \nabla \alpha) \in \partial \zeta(\dot{\alpha})+\partial_{\alpha} \mathcal{G}_{\mathrm{E}}\left(\alpha, \operatorname{dev} e_{\mathrm{el}}\right)$.

Definition 5.2 (Weak solution to (2.16a,b)-(3.1)-(3.2).). The quadruple $(u, \pi, \alpha, \phi)$ is called a weak solution to the initial-boundary-value problem (2.16a,b)-(3.1)-(3.2) with the data (3.3) provided again the data satisfies (5.1) and 5.7 a,d) holds together with

$$
\pi \in H^{1}\left(I ; L^{2}\left(\Omega_{\mathrm{S}} ; \mathbb{R}_{\mathrm{sym}}^{3 \times 3}\right)\right) \quad \text { and } \quad \alpha \in L^{\infty}\left(I ; H^{1}\left(\Omega_{\mathrm{S}}\right)\right) \cap H^{1}\left(I ; L^{2}\left(\Omega_{\mathrm{S}}\right)\right)
$$

and the integral identity (5.3a) holds with $\sigma=\sigma_{\mathrm{sph}}+\sigma_{\mathrm{dev}}$ from (3.1b,c), also (5.3b) holds with $Q$ and $\Omega$ replaced respectively $Q_{\mathrm{S}}$ and $\Omega_{\mathrm{S}}$, furthermore (5.3c) holds, and also (2.10b) holds a.e. on $Q_{\mathrm{S}}$, and eventually $u(0)=u_{0}$ holds a.e. on $\Omega$, and $\pi(0)=\pi_{0}$ and $\alpha(0)=\alpha_{0}$ hold a.e. on $\Omega_{\mathrm{S}}$. 
Sketched proof of Proposition 3.1. Like (5.4) but now with (3.3) taken into account, we have

$$
\begin{gathered}
\int_{\Omega} \frac{\varrho}{2}\left|\dot{u}_{\varepsilon}(t)\right|^{2}+\frac{3}{2} K_{\mathrm{E}}\left|\operatorname{sph} e_{\mathrm{el}, \varepsilon}(t)\right|^{2}+\frac{1}{2} \varrho\left|\omega \cdot\left(x+u_{\varepsilon}(t)\right)\right|^{2} \mathrm{~d} x+\int_{\mathbb{R}^{3}} \frac{1}{8 \pi g}\left|\nabla \phi_{\varepsilon}(t)\right|^{2} \mathrm{~d} x \\
+\int_{\Omega_{\mathrm{S}}} \mathcal{G}_{\mathrm{E}}\left(\alpha_{\varepsilon}(t), \operatorname{dev} e_{\mathrm{el}, \varepsilon}(t)\right)+\frac{\kappa}{2}\left|\nabla \alpha_{\varepsilon}(t)\right|^{2} \mathrm{~d} x \\
\quad+\varepsilon \int_{\Omega_{\mathrm{F}}} \mathcal{G}_{\mathrm{E}}\left(\alpha_{\varepsilon}(t), \operatorname{dev} e_{\mathrm{el}, \varepsilon}(t)\right)+\frac{\kappa}{2}\left|\nabla \alpha_{\varepsilon}(t)\right|^{2} \mathrm{~d} x \\
\quad+\int_{0}^{t} \int_{\Omega} \mathbb{D}_{\mathrm{KV}} \dot{e}_{\mathrm{el}, \varepsilon}: \dot{e}_{\mathrm{el}, \varepsilon} \mathrm{d} x \mathrm{~d} t+\int_{0}^{t} \int_{\Omega_{\mathrm{S}}} \mathbb{D}_{\mathrm{MX}} \dot{\pi}_{\varepsilon}: \dot{\pi}_{\varepsilon}+\dot{\alpha}_{\varepsilon} \eta^{\prime}\left(\dot{\alpha}_{\varepsilon}\right) \mathrm{d} x \mathrm{~d} t \\
+\int_{0}^{t} \int_{\Omega_{\mathrm{F}}} \frac{1}{\varepsilon} \mathbb{D}_{\mathrm{MX}} \dot{\pi}_{\varepsilon}: \dot{\pi}_{\varepsilon}+\varepsilon \dot{\alpha}_{\varepsilon} \eta^{\prime}\left(\dot{\alpha}_{\varepsilon}\right) \mathrm{d} x \mathrm{~d} t \leq \int_{\Omega}-\varrho \nabla \phi_{\varepsilon}(t) \cdot u_{\varepsilon}(t)-\varrho \phi_{\varepsilon}(t) \mathrm{d} x \\
\quad+\int_{\mathbb{R}^{3}} \varrho_{\text {ext }}(t) \phi_{\varepsilon}(t) \mathrm{d} x-\int_{0}^{t} \int_{\mathbb{R}^{3}} \dot{\varrho}_{\text {ext }} \phi_{\varepsilon} \mathrm{d} x \mathrm{~d} t+E_{0}
\end{gathered}
$$

with $E_{0}$ again from (5.5), relying that $\varepsilon \leq 1$. By the Gronwall-inequality arguments like used for (5.4), we obtain the a-priori estimates $(5.7 \mathrm{~b}, \mathrm{~d})$ now for $\left(\pi_{\varepsilon}, \phi_{\varepsilon}\right)$ instead of $\left(\pi_{k}, \phi_{k}\right)$ and also

$$
\begin{aligned}
& \left\|u_{\varepsilon}\right\|_{W^{1, \infty}\left(I ; L^{2}\left(\Omega ; \mathbb{R}^{3}\right)\right) \cap H^{1}\left(I ; H^{1}\left(\Omega_{\mathrm{S}} ; \mathbb{R}^{3}\right)\right)} \leq C \\
& \left\|\alpha_{\varepsilon}\right\|_{L^{\infty}\left(I ; H^{1}\left(\Omega_{\mathrm{S}}\right)\right) \cap H^{1}\left(I ; L^{2}\left(\Omega_{\mathrm{S}}\right)\right)} \leq C, \\
& \left\|\dot{\pi}_{\varepsilon}\right\|_{L^{2}\left(Q_{\mathrm{F}} ; \mathbb{R}^{3 \times 3}\right)} \leq \sqrt{\varepsilon} C, \\
& \left\|\dot{\alpha}_{\varepsilon}\right\|_{L^{2}\left(Q_{\mathrm{F}} ; \mathbb{R}^{3 \times 3}\right)} \leq C / \sqrt{\varepsilon}, \quad \text { and } \\
& \left\|\nabla \alpha_{\varepsilon}\right\|_{L^{\infty}\left(I ; L^{2}\left(\Omega_{\mathrm{F}} ; \mathbb{R}^{3}\right)\right)} \leq C / \sqrt{\varepsilon}
\end{aligned}
$$

Therefore, for a subsequence, we have

$$
\begin{array}{ll}
u_{\varepsilon} \rightarrow u & \text { weakly* in } W^{1, \infty}\left(I ; L^{2}\left(\Omega ; \mathbb{R}^{3}\right)\right) \cap H^{1}\left(I ; H^{1}\left(\Omega ; \mathbb{R}^{3}\right)\right), \\
\pi_{\varepsilon} \rightarrow \pi & \text { weakly in } H^{1}\left(I ; L^{2}\left(\Omega ; \mathbb{R}_{\mathrm{sym}}^{3 \times 3}\right)\right), \\
\left.\pi_{\varepsilon}\right|_{Q_{\mathrm{F}}} \rightarrow 0 & \text { strongly in } H^{1}\left(I ; L^{2}\left(\Omega_{\mathrm{F}} ; \mathbb{R}_{\mathrm{sym}}^{3 \times 3}\right)\right), \\
\left.\alpha_{\varepsilon}\right|_{Q_{\mathrm{S}}} \rightarrow \alpha & \text { weakly* in } L^{\infty}\left(I ; H^{1}\left(\Omega_{\mathrm{S}}\right)\right) \cap H^{1}\left(I ; L^{2}\left(\Omega_{\mathrm{S}}\right)\right), \\
\phi_{\varepsilon} \rightarrow \phi & \text { weakly* in } L^{\infty}\left(I ; H^{1}\left(\mathbb{R}^{3}\right)\right), \text { and also } \\
e_{\mathrm{el}, \varepsilon} \rightarrow e_{\mathrm{el}} & \text { strongly in } L^{2}\left(Q ; \mathbb{R}_{\mathrm{sym}}^{3 \times 3}\right) .
\end{array}
$$

For (5.14C), we used $\dot{\pi}_{\varepsilon} \rightarrow 0$ strongly in $L^{2}\left(Q_{\mathrm{F}} ; \mathbb{R}^{3 \times 3}\right)$ due to (5.13c) together with the assumption $\pi_{0}=0$ on $\Omega_{\mathrm{F}}$. For (5.14f), like (5.8) when taking into account that $\pi=0$ on $Q_{\mathrm{F}}$, we have

$$
\begin{aligned}
& \int_{\Omega} \frac{1}{2} \mathbb{D}_{\mathrm{KV}}\left(e_{\mathrm{el}, \varepsilon}(t)-e_{\mathrm{el}}(t)\right):\left(e_{\mathrm{el}, \varepsilon}(t)-e_{\mathrm{el}}(t)\right) \mathrm{d} x \\
& \begin{array}{l}
\leq \int_{\Omega} \frac{1}{2} \mathbb{D}_{\mathrm{KV}}\left(e_{\mathrm{el}, \varepsilon}(t)-e_{\mathrm{el}}(t)\right):\left(e_{\mathrm{el}, \varepsilon}(t)-e_{\mathrm{el}}(t)\right) \mathrm{d} x \\
\quad+\int_{\Omega_{\mathrm{S}}} \frac{1}{2} \mathbb{D}_{\mathrm{MX}}\left(\pi_{\varepsilon}(t)-\pi(t)\right):\left(\pi_{\varepsilon}(t)-\pi(t)\right) \mathrm{d} x+\int_{\Omega_{\mathrm{F}}} \frac{1}{2 \varepsilon} \mathbb{D}_{\mathrm{MX}} \pi_{\varepsilon}(t): \pi_{\varepsilon}(t) \mathrm{d} x \\
\quad+\int_{0}^{t} \int_{\Omega} K_{\mathrm{E}}\left|\operatorname{sph}\left(e_{\mathrm{el}, \varepsilon}-e_{\mathrm{el}}\right)\right|^{2}+\left(\partial_{e} \mathcal{G}_{\mathrm{E}, \varepsilon}\left(\alpha_{\varepsilon}, e_{\mathrm{el}, \varepsilon}\right)-\partial_{e} \mathcal{G}_{\mathrm{E}, \varepsilon}\left(\alpha_{\varepsilon}, e_{\mathrm{el}}\right)\right):\left(e_{\mathrm{el}, \varepsilon}-e_{\mathrm{el}}\right) \mathrm{d} x \mathrm{~d} t \\
\left.=\int_{0}^{t}\left(\int_{\Omega}-\left(\varrho \ddot{u}_{\varepsilon}+f_{\varepsilon}\right) \cdot\left(u_{\varepsilon}-u\right)-\left(\mathbb{D}_{\mathrm{KV}} \dot{e}_{\mathrm{el}}+K_{\mathrm{E}} \mathrm{sph} e_{\mathrm{el}}+\partial_{e} \mathcal{G}_{\mathrm{E}, \varepsilon}\left(\alpha_{\varepsilon}, e_{\mathrm{el}}\right)\right): e_{\mathrm{el}}\right) \mathrm{d} x-\int_{\Omega_{\mathrm{S}}} \mathbb{D}_{\mathrm{MX}} \dot{\pi}:\left(\pi_{\varepsilon}-\pi\right) \mathrm{d} x\right) \mathrm{d} t \\
=\int_{0}^{t}\left(\int_{\Omega} \varrho \dot{u}_{\varepsilon} \cdot\left(\dot{u}_{\varepsilon}-\dot{u}\right)-f_{\varepsilon} \cdot\left(u_{\varepsilon}-u\right)+\left(\partial_{e} \mathcal{G}_{\mathrm{E}, \varepsilon}\left(\alpha, e_{\mathrm{el}}\right)-\partial_{e} \mathcal{G}_{\mathrm{E}, \varepsilon}\left(\alpha_{\varepsilon}, e_{\mathrm{el}}\right)\right.\right. \\
\left.-\mathbb{D}_{\mathrm{KV}} \dot{e}_{\mathrm{el}}-K_{\mathrm{E}} \mathrm{sph} e_{\mathrm{el}}-\partial_{e} \mathcal{G}_{\mathrm{E}, \varepsilon}\left(\alpha, e_{\mathrm{el}}\right)\right):\left(e_{\mathrm{el}, \varepsilon}-e_{\mathrm{el}}\right) \mathrm{d} x \\
\left.\quad-\int_{\Omega_{\mathrm{S}}} \mathbb{D}_{\mathrm{MX}} \dot{\pi}:\left(\pi_{\varepsilon}-\pi\right) \mathrm{d} x\right) \mathrm{d} t-\int_{\Omega} \varrho \dot{u}_{\varepsilon}(t) \cdot\left(u_{\varepsilon}(t)-u(t)\right) \mathrm{d} x \rightarrow 0
\end{array}
\end{aligned}
$$


with $f_{\varepsilon}=\varrho\left(\omega \times\left(\omega \times\left(x+u_{\varepsilon}\right)\right)+2 \omega \times \dot{u}_{\varepsilon}+\nabla \phi_{\varepsilon}\right)$ bounded in $L^{2}\left(Q ; \mathbb{R}^{3}\right)$ and where we now used the continuity of the Nemytskil mapping induced by $\partial_{e} \mathcal{G}_{\mathrm{E}, \varepsilon}\left(\cdot, e_{\mathrm{el}}\right)=\partial_{e} \mathcal{G}_{\mathrm{E}}\left(\cdot, e_{\mathrm{el}}\right)$ on $Q_{\mathrm{S}}$ while, on $Q_{\mathrm{F}}$, we use that simply $\partial_{e} \mathcal{G}_{\mathrm{E}, \varepsilon}\left(\alpha_{\varepsilon}, e_{\mathrm{el}}\right)=\varepsilon \partial_{e} \mathcal{G}_{\mathrm{E}}\left(\alpha_{\varepsilon}, e_{\mathrm{el}}\right) \rightarrow 0$ strongly in $L^{2}\left(Q_{\mathrm{F}}\right)$; recall the scaling (3.3).

The limit passage towards the weak solution in the sense of Definition 5.2 is then simple by the continuity with respect to the convergences (5.14). More details deserve only the limit passage in (5.3b) which, written for $\left(u_{\varepsilon}, \pi_{\varepsilon}, \alpha_{\varepsilon}\right)$ and omitting the terms $\varepsilon \int_{Q_{\mathrm{S}}} \eta\left(\dot{\alpha}_{\varepsilon}\right) \mathrm{d} x \mathrm{~d} t \geq 0$ and $\varepsilon \int_{\Omega_{\mathrm{F}}} \frac{\kappa}{2}\left|\nabla \alpha_{\varepsilon}(T)\right|^{2} \mathrm{~d} x \geq 0$, reads as:

$$
\begin{gathered}
\int_{Q_{\mathrm{S}}}\left(v-\dot{\alpha}_{\varepsilon}\right) \partial_{\alpha} \mathcal{G}_{\mathrm{E}, \varepsilon}\left(\alpha_{\varepsilon}, \operatorname{dev}\left(e\left(u_{\varepsilon}\right)-\pi_{\varepsilon}\right)\right)+\kappa \nabla \alpha_{\varepsilon} \cdot \nabla v+\eta(v) \mathrm{d} x \mathrm{~d} t+\int_{\Omega_{\mathrm{S}}} \frac{\kappa}{2}\left|\nabla \alpha_{0}\right|^{2} \mathrm{~d} x \\
+\varepsilon\left(\int_{Q_{\mathrm{F}}}\left(v-\dot{\alpha}_{\varepsilon}\right) \partial_{\alpha} \mathcal{G}_{\mathrm{E}, \varepsilon}\left(\alpha_{\varepsilon}, \operatorname{dev}\left(e\left(u_{\varepsilon}\right)-\pi_{\varepsilon}\right)\right)+\kappa \nabla \alpha_{\varepsilon} \cdot \nabla v+\eta(v) \mathrm{d} x \mathrm{~d} t\right. \\
\left.+\int_{\Omega_{\mathrm{F}}} \frac{\kappa}{2}\left|\nabla \alpha_{0}\right|^{2} \mathrm{~d} x\right) \geq \int_{Q_{\mathrm{S}}} \eta\left(\dot{\alpha}_{\varepsilon}\right) \mathrm{d} x \mathrm{~d} t+\int_{\Omega_{\mathrm{S}}} \frac{\kappa}{2}\left|\nabla \alpha_{\varepsilon}(T)\right|^{2} \mathrm{~d} x .
\end{gathered}
$$

Now we use

$$
\left|\varepsilon \int_{Q_{\mathrm{F}}} \dot{\alpha}_{\varepsilon} \partial_{\alpha} \mathcal{G}_{\mathrm{E}, \varepsilon}\left(\alpha_{\varepsilon}, e_{\mathrm{el}, \varepsilon}\right) \mathrm{d} x \mathrm{~d} t\right| \leq \varepsilon C\left\|\dot{\alpha}_{\varepsilon}\right\|_{L^{2}\left(Q_{\mathrm{F}}\right)}\left\|1+\left|e_{\mathrm{el}, \varepsilon}\right|\right\|_{L^{2}(Q)}=\mathscr{O}(\sqrt{\varepsilon}) \rightarrow 0
$$

due to (5.13d), and

$$
\left.\left|\varepsilon \int_{Q_{\mathrm{F}}} \kappa \nabla \alpha_{\varepsilon} \cdot \nabla v \mathrm{~d} x \mathrm{~d} t\right| \leq \varepsilon\left(\sup _{\Omega} \kappa\right)\right)\left\|\nabla \alpha_{\varepsilon}\right\|_{L^{2}\left(Q_{\mathrm{F}} ; \mathbb{R}^{3}\right)}\|\nabla v\|_{L^{2}\left(Q ; \mathbb{R}^{3}\right)}=\mathscr{O}(\sqrt{\varepsilon}) \rightarrow 0
$$

due to (5.13e). In the limit we thus obtain (5.3b) on $\Omega_{\mathrm{S}}$.

Altogether, we proved that the limit $(u, \pi, \alpha, \phi)$ solves the initial-boundary-value problem (3.1) - (2.16a,b)-(3.2) in the sense of Definition 5.2.

In addition, this solution conserves energy. This can be shown again by using that $\sqrt{\varrho} \ddot{u}$ in duality with $\sqrt{\varrho} \dot{u}$ so that (5.9a) holds, and that $\operatorname{div}(\kappa \nabla \alpha) \in L^{2}\left(Q_{\mathrm{S}}\right)$ and $\dot{\alpha} \in L^{2}\left(Q_{\mathrm{S}}\right)$ so that also (5.9b) holds but now with $Q_{\mathrm{S}}$ and $\Omega_{\mathrm{S}}$ instead of $Q$ and $\Omega$, respectively.

To prove (3.4), we use (5.4) written for $\left(u_{\varepsilon}, \pi_{\varepsilon}, \alpha_{\varepsilon}, \phi_{\varepsilon}\right)$ with $t=T$ and the scaling (3.2), namely

$$
\begin{aligned}
& \limsup _{\varepsilon \rightarrow 0} \int_{Q_{\mathrm{F}}} \frac{1}{\varepsilon} \mathbb{D}_{\mathrm{MX}} \dot{\pi}_{\varepsilon}: \dot{\pi}_{\varepsilon}+\varepsilon \dot{\alpha}_{\varepsilon} \zeta\left(\dot{\alpha}_{\varepsilon}\right) \mathrm{d} x \mathrm{~d} t \\
& \leq \lim _{\varepsilon \rightarrow 0} \int_{\mathbb{R}^{3}} \varrho_{\text {ext }}(T) \phi_{\varepsilon}(T) \mathrm{d} x-\int_{\Omega} \varrho \nabla \phi_{\varepsilon}(T) \cdot u_{\varepsilon}(T)-\varrho \phi_{\varepsilon}(T) \mathrm{d} x-\int_{0}^{T} \int_{\mathbb{R}^{3}} \dot{\varrho}_{\text {ext }} \phi_{\varepsilon} \mathrm{d} x \mathrm{~d} t \\
& -\int_{Q}\left(\omega \times\left(\omega \times\left(x+u_{\varepsilon}\right) \cdot \dot{u}_{\varepsilon} \mathrm{d} x \mathrm{~d} t-\int_{\Omega_{\mathrm{F}}} \varepsilon \mathcal{G}_{\mathrm{E}}\left(\alpha_{\varepsilon}(T), e_{\mathrm{el}, \varepsilon}(T)\right) \mathrm{d} x\right.\right. \\
& -\liminf _{\varepsilon \rightarrow 0}\left(\int_{\Omega} \frac{\varrho}{2}\left|\dot{u}_{\varepsilon}(T)\right|^{2}+\frac{3}{2} K_{\mathrm{E}}\left|\operatorname{sph} e_{\mathrm{el}, \varepsilon}(T)\right|^{2} \mathrm{~d} x\right. \\
& +\int_{\Omega_{\mathrm{S}}} \mathcal{G}_{\mathrm{E}}\left(\alpha_{\varepsilon}(T), e_{\mathrm{el}, \varepsilon}(T)\right)+\frac{\kappa}{2}\left|\nabla \alpha_{\varepsilon}(T)\right|^{2} \mathrm{~d} x+\int_{\mathbb{R}^{3}} \frac{1}{8 \pi g}\left|\nabla \phi_{\varepsilon}(T)\right|^{2} \mathrm{~d} x \\
& \left.+\int_{Q} \mathbb{D}_{\mathrm{KV}} \dot{e}_{\mathrm{el}, \varepsilon}: \dot{e}_{\mathrm{el}, \varepsilon} \mathrm{d} x \mathrm{~d} t+\int_{Q_{\mathrm{S}}} \mathbb{D}_{\mathrm{MX}} \dot{\pi}_{\varepsilon}: \dot{\pi}_{\varepsilon}+\dot{\alpha}_{\varepsilon} \eta^{\prime}\left(\dot{\alpha}_{\varepsilon}\right) \mathrm{d} x \mathrm{~d} t\right)+E_{0} \\
& \leq \int_{\mathbb{R}^{3}} \varrho_{\text {ext }}(T) \phi(T)-\frac{1}{8 \pi g}|\nabla \phi(T)|^{2} \mathrm{~d} x-\int_{\Omega} \varrho \nabla \phi(T) \cdot u(T)-\varrho \phi(T) \mathrm{d} x \\
& -\int_{0}^{T} \int_{\mathbb{R}^{3}} \dot{\varrho}_{\mathrm{ext}} \phi \mathrm{d} x \mathrm{~d} t-\int_{Q} \mathbb{D}_{\mathrm{KV}} \dot{e}_{\mathrm{el}}: \dot{e}_{\mathrm{el}}+(\omega \times(\omega \times(x+u) \cdot \dot{u} \mathrm{~d} x \mathrm{~d} t \\
& -\int_{\Omega} \frac{\varrho}{2}|\dot{u}(T)|^{2}+\frac{3}{2} K_{\mathrm{E}}\left|\operatorname{sph} e_{\mathrm{el}}(T)\right|^{2} \mathrm{~d} x-\int_{\Omega_{\mathrm{S}}} \mathcal{G}_{\mathrm{E}}\left(\alpha(T), e_{\mathrm{el}}(T)\right)+\frac{\kappa}{2}|\nabla \alpha(T)|^{2} \mathrm{~d} x \\
& -\int_{Q_{\mathrm{S}}} \mathbb{D}_{\mathrm{MX}} \dot{\pi}: \dot{\pi}+\dot{\alpha} \eta^{\prime}(\dot{\alpha}) \mathrm{d} x \mathrm{~d} t+E_{0}=0
\end{aligned}
$$


where we again used the notation (2.9) and $E_{0}$ from (5.5) now with $\kappa=0$ on $\Omega_{\mathrm{F}}$. The first inequality arises from "forgetting" the term $\frac{\kappa}{2}\left|\nabla \alpha_{\varepsilon}(T)\right|^{2}$ on $\Omega_{\mathrm{F}}$, while the second inequality is by weak lower semicontinuity. The last equality in (5.17) expresses the energy conservation for the limit system, discussed already above. Thus (3.4) is proved.

Remark 5.3 (Strong convergence of total strains). From (5.15), one can even see that also $\pi_{\varepsilon} \rightarrow \pi$ strongly so that, together with (5.14f) ), even the total strain $e\left(u_{\varepsilon}\right)=e_{\mathrm{el}, \varepsilon}+\pi_{\varepsilon}$ converges strongly in $L^{2}\left(Q ; \mathbb{R}_{\mathrm{sym}}^{3 \times 3}\right)$. We did not need this additional result in the above convergence proof, however.

Remark 5.4 (Damage flow rule almost everywhere). Since $\partial \zeta(\dot{\alpha})$ is bounded in $L^{2}(Q)$ in our model, by comparison, we have also $\operatorname{div}(\kappa \nabla \alpha) \in \partial \eta(\dot{\alpha})+\partial_{\alpha} \mathcal{G}_{\mathrm{E}}\left(\alpha, \operatorname{dev} e_{\mathrm{el}}\right)$ bounded in $L^{2}(Q)$, cf. (3.1e). Since also $\dot{\alpha} \in L^{2}(Q)$, the formula $\int_{Q} \dot{\alpha} \operatorname{div}(\kappa \nabla \alpha) \mathrm{d} x \mathrm{~d} t=\frac{1}{2} \int_{\Omega} \kappa\left|\nabla \alpha_{0}\right|^{2}-\kappa|\nabla \alpha(T)|^{2} \mathrm{~d} x$ rigorously holds, and we can write (5.3b) in as the original inequality (2.11) holding a.e. on $Q$. The integral form (5.3b) is however suitable for the limit passages, in contrast to (2.11).

Definition 5.5. Assuming, beside (5.1), also $e_{\mathrm{el}, 0}=e\left(u_{0}\right)-\pi_{0} \in H^{1}\left(\Omega ; \mathbb{R}^{3 \times 3}\right)$ and $v_{0} \in$ $H^{2}\left(\Omega ; \mathbb{R}^{3}\right)$, then the weak solution to the system $(3.1 \mathrm{a}, \mathrm{d}-\mathrm{f})-(2.16 \mathrm{a}, \mathrm{b})-(3.2)$ with (4.1) is understood as a five-tuple $\left(u, \sigma_{\mathrm{sph}}, \sigma_{\mathrm{dev}}, \pi, \phi\right) \in W^{1, \infty}\left(I ; L^{2}\left(\Omega ; \mathbb{R}^{3}\right)\right) \times L^{2}\left(Q ; \mathbb{R}_{\mathrm{sph}}^{3 \times 3}\right) \times L^{2}\left(Q ; \mathbb{R}_{\mathrm{dev}}^{3 \times 3}\right) \times$ $H^{1}\left(I ; L^{2}\left(\Omega_{\mathrm{S}} ; \mathbb{R}_{\mathrm{sym}}^{3 \times 3}\right)\right) \times W^{1, \infty}\left(I ; H^{1}\left(\mathbb{R}^{3}\right)\right)$ if the integral identity

$$
\int_{Q_{\mathrm{S}}} \sigma_{\mathrm{dev}}: \operatorname{dev} e(v) \mathrm{d} x \mathrm{~d} t+\int_{Q} \sigma_{\mathrm{sph}}: \operatorname{sph} e(v)-\varrho \dot{u} \cdot \dot{v}+f \cdot v \mathrm{~d} x \mathrm{~d} t=\int_{\Omega} \varrho v_{0} \cdot v(0, \cdot) \mathrm{d} x
$$

with $f$ from (2.10a) holds for any $v \in H^{1}\left(Q ; \mathbb{R}^{3}\right)$ with $v(T)=0$, further (3.1d) hold a.e. on $Q_{\mathrm{S}}$ and (4.1) relating $\left(\sigma_{\mathrm{dev}}, \sigma_{\mathrm{sph}}\right)$ with $(u, \pi)$ hold a.e. on $Q$, and also the initial conditions $u(0, \cdot)=u_{0}$ and $\pi(0, \cdot)=\pi_{0}$ hold a.e. on $\Omega$.

Note that, controlling $\sigma_{\mathrm{sph}}$ and $\sigma_{\mathrm{dev}}$ in the above definition, we have implicitly also included the information

$$
\left.u\right|_{Q_{\mathrm{S}}} \in H^{1}\left(I ; H^{1}\left(\Omega ; \mathbb{R}^{3}\right)\right) \quad \text { and } \quad u \in L^{2}\left(I ; L_{\mathrm{div}}^{2}\left(\Omega ; \mathbb{R}^{3}\right)\right)
$$

with $L_{\text {div }}^{2}\left(\Omega ; \mathbb{R}^{3}\right)=\left\{u \in L^{2}\left(\Omega ; \mathbb{R}^{3}\right) ; \operatorname{div} u \in L^{2}(\Omega)\right\}$, while the strain $e(u)$ is not defined in the fluidic regions $Q_{\mathrm{F}}$. Also one has $e(u)-\pi \in H^{1}\left(I ; L^{2}\left(\Omega_{\mathrm{S}} ; \mathbb{R}_{\mathrm{sym}}^{3 \times 3}\right)\right)$.

Sketched proof of Proposition 4.1. We first prove a certain regularity by differentiating in time the system (3.1 a,d) with (4.1) written for the solution obtained in Proposition 2.1 and denoted now as $\left(u_{\varepsilon}, \pi_{\varepsilon}, \alpha_{\varepsilon}, \phi_{\varepsilon}\right)$, and employing the test by $\left(\ddot{u}_{\varepsilon}, \ddot{\pi}_{\varepsilon}\right)$. Taking into account the scaling (4.2) and using again the orthogonality for the Coriolis force (now in terms of accelerations) as $(2 \varrho \omega \times \ddot{u}) \cdot \ddot{u}=0$, this gives

$$
\begin{aligned}
& \int_{\Omega} \frac{\varrho}{2}\left|\ddot{u}_{\varepsilon}(t)\right|^{2}+\frac{3}{2} K_{\mathrm{E}}\left|\operatorname{sph} \dot{e}_{\mathrm{el}, \varepsilon}(t)\right|^{2} \mathrm{~d} x \\
& +\int_{0}^{t} \int_{\Omega_{\mathrm{S}}} \mathbb{D}_{\mathrm{KV}} \ddot{e}_{\mathrm{el}, \varepsilon}: \ddot{e}_{\mathrm{el}, \varepsilon}+\mathbb{D}_{\mathrm{MX}} \ddot{\pi}_{\varepsilon}: \ddot{\pi}_{\varepsilon} \mathrm{d} x \mathrm{~d} t+\varepsilon \int_{0}^{t} \int_{\Omega_{\mathrm{F}}} \mathbb{D}_{\mathrm{KV}} e\left(\ddot{u}_{\varepsilon}\right): e\left(\ddot{u}_{\varepsilon}\right) \mathrm{d} x \mathrm{~d} t \\
& =\int_{\Omega} \frac{\varrho}{2}\left|\ddot{u}_{\varepsilon}(0)\right|^{2}+\frac{3}{2} K_{\mathrm{E}}\left|\operatorname{sph} \dot{e}_{\mathrm{el}, \varepsilon}(0)\right|^{2} \mathrm{~d} x-\int_{0}^{t} \int_{\Omega} \varrho\left(\omega \times\left(\omega \times\left(x+\dot{u}_{\varepsilon}\right)\right)+\nabla \dot{\phi}_{\varepsilon}\right) \cdot \ddot{u}_{\varepsilon} \\
& -\int_{0}^{t} \int_{\Omega_{\mathrm{S}}}\left(\partial_{e e}^{2} \mathcal{G}_{\mathrm{E}}\left(\alpha_{\varepsilon}, e_{\mathrm{el}, \varepsilon}\right): \operatorname{dev} \dot{e}_{\mathrm{el}, \varepsilon}+\partial_{e \alpha}^{2} \mathcal{G}_{\mathrm{E}}\left(\alpha_{\varepsilon}, e_{\mathrm{el}, \varepsilon}\right) \dot{\alpha}_{\varepsilon}\right): \operatorname{dev} \ddot{e}_{\mathrm{el}, \varepsilon} \mathrm{d} x \mathrm{~d} t \\
& \leq \frac{1}{2}\|\varrho\|_{L^{\infty}(\Omega)}\left\|\ddot{u}_{\varepsilon}(0)\right\|_{L^{2}\left(\Omega ; \mathbb{R}^{3}\right)}^{2}+\frac{3}{2} K_{\mathrm{E}}\left\|\operatorname{sph}\left(e\left(v_{0}\right)-\dot{\pi}_{0}\right)\right\|_{L^{2}\left(\Omega ; \mathbb{R}^{3 \times 3}\right)}^{2} \\
& +\|\sqrt{\varrho}\|_{L^{\infty}(\Omega)}\left\|\omega \times\left(\omega \times\left(x+\dot{u}_{\varepsilon}\right)\right)+\nabla \dot{\phi}_{\varepsilon}\right\|_{L^{2}\left(Q ; \mathbb{R}^{3}\right)}^{2}+\int_{0}^{t} \int_{\Omega} \frac{\varrho}{2}\left|\ddot{u}_{\varepsilon}(t)\right|^{2} \mathrm{~d} x \mathrm{~d} t \\
& +\frac{1}{c}\left(\left\|\partial_{e e}^{2} \mathcal{G}_{\mathrm{E}}\left(\alpha_{\varepsilon}, e_{\mathrm{el}, \varepsilon}\right)\right\|_{L^{\infty}\left(Q_{\mathrm{S}} ; \mathbb{R}^{3}\right)}^{2}\left\|e\left(\dot{u}_{\varepsilon}\right)\right\|_{L^{2}\left(Q ; \mathbb{R}^{3 \times 3}\right)}^{2}\right. \\
& \left.+\left\|\partial_{e \alpha}^{2} \mathcal{G}_{\mathrm{E}}\left(\alpha_{\varepsilon}, e_{\mathrm{el}, \varepsilon}\right)\right\|_{L^{\infty}\left(Q ; \mathbb{R}^{3 \times 3}\right)}^{2}\left\|\dot{\alpha}_{\varepsilon}\right\|_{L^{2}(Q)}^{2}\right)+\epsilon\left\|\ddot{e}_{\mathrm{el}, \varepsilon}\right\|_{L^{2}\left([0, t] \times \Omega_{\mathrm{S}} ; \mathbb{R}^{3 \times 3}\right)}^{2}
\end{aligned}
$$


with $c:=\frac{1}{2}\left\|\mathbb{D}_{\mathrm{KV}}\right\|_{L^{\infty}\left(\Omega ; \mathbb{R}^{3^{4}}\right)}$; then the last term in (15.19) can be absorbed in the left hand side. Note that we needed the assumptions (5.1d $)$ to have $\partial_{e \alpha}^{2} \mathcal{G}_{\mathrm{E}}\left(\alpha_{\varepsilon}, e_{\mathrm{el}, \varepsilon}\right)$ and $\partial_{e e}^{2} \mathcal{G}_{\mathrm{E}}\left(\alpha_{\varepsilon}, e_{\mathrm{el}, \varepsilon}\right)$ apriori bounded. We further use

$$
\ddot{u}_{\varepsilon}(0)=\frac{1}{\varrho}\left(\operatorname{div} \sigma_{0, \varepsilon}+\varrho\left(\omega \times\left(\omega \times\left(x+u_{0}\right)\right)+2 \omega \times v_{0}+\nabla \phi_{0}\right)\right) \in L^{2}\left(\Omega ; \mathbb{R}^{3}\right)
$$

with the initial stress $\sigma_{0, \varepsilon}=\sigma_{\mathrm{sph}}+\sigma_{\mathrm{dev}}$ with $\sigma_{\mathrm{sph}}$ and $\sigma_{\mathrm{dev}}$ from $(3.1 \mathrm{p}, \mathrm{c})$ with $\left(K_{\mathrm{KV}}, G_{\mathrm{KV}}\right)=$ $\left(K_{\mathrm{KV}, \varepsilon}, G_{\mathrm{KV}, \varepsilon}\right)$ from (4.2), and with $e_{\mathrm{el}}=e\left(u_{0}\right)-\pi_{0}$ and $\dot{e}_{\mathrm{el}}=e\left(v_{0}\right)-\dot{\pi}_{0}$, and $\phi_{0} \in H^{1}\left(\mathbb{R}^{3}\right)$ solving $\operatorname{div}\left(\nabla \phi_{0} /(4 \pi g)+\varrho u_{0}\right)=\varrho+\varrho_{\text {ext }}(0)$. Note that indeed $\ddot{u}_{\varepsilon}(0) \in L^{2}\left(\Omega ; \mathbb{R}^{3}\right)$ due to the assumptions $e_{\mathrm{el}, 0}=e\left(u_{0}\right)-\pi_{0} \in H^{1}\left(\Omega ; \mathbb{R}^{3 \times 3}\right)$ and $v_{0} \in H^{2}\left(\Omega ; \mathbb{R}^{3}\right)$ involved in Definition 5.5. Also note that $\dot{\pi}_{0}:=\dot{\pi}(0)$ is involved in (5.19) and implicitly also in (5.20), although we do not prescribe any initial condition on $\dot{\pi}$. Yet, from $(3.1 \mathrm{~d})$, we can read $\dot{\pi}(0)=\mathbb{D}_{\mathrm{Mx}}^{-1} \sigma_{0}$ on $\Omega_{\mathrm{S}}$ while $\dot{\pi}(0)=0$ on $\Omega_{\mathrm{F}}$ because we have now $\mathbb{D}_{\mathrm{Mx}}^{-1}=0$ on $\Omega_{\mathrm{F}}$. To estimate $\sigma_{0}$ let us realize that $\left\|\sigma_{0}\right\|_{H^{1}\left(\Omega ; \mathbb{R}^{3 \times 3}\right)} \leq\left\|\mathbb{D}_{\mathrm{KV}}\left(e\left(v_{0}\right)-\dot{\pi}(0)\right)+\partial_{e} \mathcal{G}_{\mathrm{E}}\left(\alpha_{0}, e_{\mathrm{el}, 0}\right)\right\|_{H^{1}\left(\Omega ; \mathbb{R}^{3 \times 3}\right)} \leq C+\left\|\mathbb{D}_{\mathrm{KV}} \mathbb{D}_{\mathrm{MX}}^{-1} \sigma_{0}\right\|_{H^{1}\left(\Omega ; \mathbb{R}^{3 \times 3}\right)}$ with some $C$ depending on $\left\|v_{0}\right\|_{H^{2}\left(\Omega ; \mathbb{R}^{3}\right)},\left\|\alpha_{0}\right\|_{H^{1}(\Omega)}$, and $\left\|e_{\mathrm{el}, 0}\right\|_{H^{1}\left(\Omega ; \mathbb{R}^{3 \times 3}\right)}$; note that these quantities have been supposed finite in Definition 5.5. Thus, using (5.1c), we get the desired bound on $\sigma_{0}$. Hence, $\dot{e}_{\mathrm{el}}(0)$ which occurs in (5.19) itself can be estimated.

Using Gronwall's inequality for (5.19) then yields the estimates

$$
\begin{aligned}
& \left\|u_{\varepsilon}\right\|_{W^{2, \infty}\left(I ; L^{2}\left(\Omega ; \mathbb{R}^{3}\right)\right) \cap H^{2}\left(I ; H^{1}\left(\Omega_{\mathrm{S}} ; \mathbb{R}^{3}\right)\right)} \leq C, \\
& \left\|\ddot{\pi}_{\varepsilon}\right\|_{L^{2}\left(Q_{\mathrm{S}} ; \mathbb{R}^{3 \times 3}\right)} \leq C, \quad \text { and } \\
& \left\|u_{\varepsilon}\right\|_{H^{2}\left(I ; H^{1}\left(\Omega_{\mathrm{F}} ; \mathbb{R}^{3}\right)\right)} \leq C / \sqrt{\varepsilon}
\end{aligned}
$$

which are now at disposal together with (5.2d) and (5.13b). Also we have

$$
\left\|\sigma_{\varepsilon}\right\|_{L^{2}\left(Q ; \mathbb{R}_{\mathrm{sym}}^{3 \times 3}\right)} \leq C \quad \text { with } \sigma_{\varepsilon}=\left\{\begin{array}{cl}
\varepsilon \mathbb{D}_{\mathrm{KV}} e\left(\dot{u}_{\varepsilon}\right)+3 K_{\mathrm{KV}} \mathrm{sph} e\left(u_{\varepsilon}\right) & \text { on } Q_{\mathrm{F}} \\
\mathbb{D}_{\mathrm{KV}} \dot{e}_{\mathrm{el}, \varepsilon}+\mathbb{C}_{\mathrm{E}}\left(\alpha_{\varepsilon}, e_{\mathrm{el}, \varepsilon}\right) & \text { on } Q_{\mathrm{S}}
\end{array}\right.
$$

where we again use the notation (2.9) and $e_{\mathrm{el}, \varepsilon}=e\left(u_{\varepsilon}\right)-\pi_{\varepsilon}$.

By Banach's selection principle, we consider a weakly* convergent subsequence respecting the topologies specified in $(5.21 \mathrm{a}, \mathrm{b})$ and $(5.13 \mathrm{~b})$ together with the convergence $(5.14 \mathrm{e})$ and also $\sigma_{\varepsilon} \rightarrow \sigma$ weakly in $L^{2}\left(Q ; \mathbb{R}_{\mathrm{sym}}^{3 \times 3}\right)$. Then we put naturally $\sigma_{\mathrm{sph}}:=\operatorname{sph} \sigma$ and $\sigma_{\mathrm{dev}}:=\operatorname{dev} \sigma$. As $\left\|e\left(\dot{u}_{\varepsilon}\right)\right\|_{L^{2}\left(Q_{\mathrm{F}} ; \mathbb{R}^{3 \times 3}\right)}=\mathscr{O}(1 / \sqrt{\varepsilon})$, we have $\left\|\varepsilon \mathbb{D}_{\mathrm{KV}} e\left(\dot{u}_{\varepsilon}\right)\right\|_{L^{2}\left(Q_{\mathrm{F}} ; \mathbb{R}^{3 \times 3}\right)}=\mathscr{O}(\sqrt{\varepsilon}) \rightarrow 0$ so that $\sigma=3 K_{\mathrm{KV}} \operatorname{sph} e(u)$ on $Q_{\mathrm{F}}$. To identify $\sigma=\mathbb{D}_{\mathrm{KV}} \dot{e}_{\mathrm{el}}+\mathbb{C}_{\mathrm{E}}\left(\alpha_{\varepsilon}, e_{\mathrm{el}}\right)$ in the solid regions $Q_{\mathrm{S}}$, due to nonlinearities $\mathbb{C}_{\mathrm{E}}(\alpha, \cdot)$, we again need to prove $e_{\mathrm{el}, \varepsilon} \rightarrow e_{\mathrm{el}}$ strongly in $L^{2}\left(Q_{\mathrm{S}} ; \mathbb{R}_{\mathrm{sym}}^{3 \times 3}\right)$. To this goal, one is to modify (5.15) to be used on $\Omega_{\mathrm{S}}$ rather than $\Omega$. The peculiarity is that $u_{\varepsilon}-u$ is no longer a legitimate test function because $e(u)$ is not well defined in the fluidic regions $Q_{\mathrm{F}}$. For this reason, we take some smooth $\tilde{u}_{\varepsilon}$ that will approximate $u$ strongly in $L^{\infty}\left(I ; L_{\text {div }}^{2}\left(\Omega ; \mathbb{R}^{3}\right)\right)$ and even $\left.\left.\tilde{u}_{\varepsilon}\right|_{Q_{\mathrm{S}}} \rightarrow u\right|_{Q_{\mathrm{S}}}$ strongly in $H^{1}\left(I ; H^{1}\left(\Omega ; \mathbb{R}^{3}\right)\right)$, and we can assume that this convergence is sufficiently slow so that

$$
\left\|\tilde{u}_{\varepsilon}\right\|_{L^{2}\left(I ; H^{1}\left(\Omega_{\mathrm{F}} ; \mathbb{R}^{3}\right)\right)} \leq 1 / \sqrt[4]{\varepsilon}
$$

Then, denoting $\tilde{e}_{\mathrm{el}, \varepsilon}=e\left(\tilde{u}_{\varepsilon}\right)-\pi$, we have $\tilde{e}_{\mathrm{el}, \varepsilon}(t) \rightarrow e_{\mathrm{el}}(t)$ for a.a. $t \in I$ and we can write

$$
\begin{aligned}
\limsup _{\varepsilon \rightarrow 0} \int_{\Omega_{\mathrm{S}}} & \frac{1}{4} \mathbb{D}_{\mathrm{KV}}\left(e_{\mathrm{el}, \varepsilon}(t)-e_{\mathrm{el}}(t)\right):\left(e_{\mathrm{el}, \varepsilon}(t)-e_{\mathrm{el}}(t)\right) \mathrm{d} x \\
\leq \limsup _{\varepsilon \rightarrow 0} \int_{\Omega_{\mathrm{S}}} & \frac{1}{2} \mathbb{D}_{\mathrm{KV}}\left(e_{\mathrm{el}, \varepsilon}(t)-\tilde{e}_{\mathrm{el}, \varepsilon}(t)\right):\left(\tilde{e}_{\mathrm{el}, \varepsilon}(t)-e_{\mathrm{el}, \varepsilon}(t)\right) \mathrm{d} x \\
\quad & \quad \lim _{\varepsilon \rightarrow 0} \int_{\Omega_{\mathrm{S}}} \frac{1}{2} \mathbb{D}_{\mathrm{KV}}\left(\tilde{e}_{\mathrm{el}, \varepsilon}(t)-e_{\mathrm{el}}(t)\right):\left(\tilde{e}_{\mathrm{el}, \varepsilon}(t)-e_{\mathrm{el}}(t)\right) \mathrm{d} x
\end{aligned}
$$




$$
\begin{aligned}
\leq \limsup _{\varepsilon \rightarrow 0}\left(\int _ { \Omega _ { \mathrm { S } } } \left(\frac{1}{2} \mathbb{D}_{\mathrm{KV}}\left(e_{\mathrm{el}, \varepsilon}(t)-\tilde{e}_{\mathrm{el}, \varepsilon}(t)\right):\left(e_{\mathrm{el}, \varepsilon}(t)-\tilde{e}_{\mathrm{el}, \varepsilon}(t)\right)\right.\right. \\
\left.+\frac{1}{2} \mathbb{D}_{\mathrm{MX}}\left(\pi_{\varepsilon}(t)-\pi(t)\right):\left(\pi_{\varepsilon}(t)-\pi(t)\right)\right) \mathrm{d} x+\int_{0}^{t} \int_{\Omega} K_{\mathrm{E}}\left|\operatorname{sph}\left(e_{\mathrm{el}, \varepsilon}-\tilde{e}_{\mathrm{el}, \varepsilon}\right)\right|^{2} \mathrm{~d} x \mathrm{~d} t \\
\left.\quad+\int_{0}^{t} \int_{\Omega_{\mathrm{S}}}\left(\partial_{e} \mathcal{G}_{\mathrm{E}}\left(\alpha_{\varepsilon}, e_{\mathrm{el}, \varepsilon}\right)-\partial_{e} \mathcal{G}_{\mathrm{E}}\left(\alpha_{\varepsilon}, \tilde{e}_{\mathrm{el}, \varepsilon}\right)\right):\left(e_{\mathrm{el}, \varepsilon}-\tilde{e}_{\mathrm{el}, \varepsilon}\right) \mathrm{d} x \mathrm{~d} t\right) \\
\leq \lim _{\varepsilon \rightarrow 0}\left(\int_{\Omega_{\mathrm{F}}} \frac{\varepsilon}{2} \mathbb{D}_{\mathrm{KV}} e\left(u_{0}\right) \mathrm{d} x-\int_{0}^{t} \int_{\Omega_{\mathrm{F}}} \varepsilon \mathbb{D}_{\mathrm{KV}} e\left(\dot{u}_{\varepsilon}\right): e\left(\tilde{u}_{\varepsilon}\right) \mathrm{d} x \mathrm{~d} t\right. \\
\quad-\int_{0}^{t} \int_{\Omega}\left(\varrho \ddot{u}_{\varepsilon}+f_{\varepsilon}\right) \cdot\left(u_{\varepsilon}-\tilde{u}_{\varepsilon}\right)-\int_{0}^{t} \int_{\Omega_{\mathrm{S}}} \mathbb{D}_{\mathrm{MX}} \dot{\pi}:\left(\pi_{\varepsilon}-\pi\right) \\
\left.+\quad\left(\mathbb{D}_{\mathrm{KV}} \dot{\tilde{e}}_{\mathrm{el}, \varepsilon}+K_{\mathrm{E}} \mathrm{sph} \tilde{e}_{\mathrm{el}, \varepsilon}+\partial_{e} \mathcal{G}_{\mathrm{E}}\left(\alpha_{\varepsilon}, \tilde{e}_{\mathrm{el}, \varepsilon}\right)\right):\left(e_{\mathrm{el}, \varepsilon}-\tilde{e}_{\mathrm{el}, \varepsilon}\right) \mathrm{d} x \mathrm{~d} t\right)=0,
\end{aligned}
$$

where the third inequality have arisen by "forgetting" the nonnegative term $\int_{\Omega_{\mathrm{F}}} \varepsilon \mathbb{D}_{\mathrm{KV}} e\left(u_{\varepsilon}(T)\right)$ : $e\left(u_{\varepsilon}(T)\right) \mathrm{d} x$. Note the we used (5.22) together with (5.21c) for the estimate

$$
\begin{aligned}
& \left|\int_{0}^{t} \int_{\Omega_{\mathrm{F}}} \varepsilon \mathbb{D}_{\mathrm{KV}} e\left(\dot{u}_{\varepsilon}\right): e\left(\tilde{u}_{\varepsilon}\right) \mathrm{d} x \mathrm{~d} t\right| \leq \varepsilon\left\|\mathbb{D}_{\mathrm{KV}}\right\|_{L^{\infty}\left(\Omega ; \mathbb{R}^{3^{4}}\right)} \times \\
& \quad \times\left\|e\left(\dot{u}_{\varepsilon}\right)\right\|_{L^{2}\left(Q_{\mathrm{F}} ; \mathbb{R}^{3 \times 3}\right)}\left\|e\left(\tilde{u}_{\varepsilon}\right)\right\|_{\left.L^{2}\left(Q_{\mathrm{F}} ; \mathbb{R}^{3 \times 3}\right)\right)}=\varepsilon \mathscr{O}\left(\frac{1}{\sqrt[3 / 4]{\varepsilon}}\right)=\mathscr{O}(\sqrt[4]{\varepsilon}) \rightarrow 0 .
\end{aligned}
$$

From (5.23), we thus have $\left.\left.e_{\mathrm{el}, \varepsilon}\right|_{Q_{\mathrm{S}}}(t) \rightarrow e_{\mathrm{el}}\right|_{Q_{\mathrm{S}}}(t)$ at a.a. $t \in I$. Then, instead of (5.14f) , by the Lebesgue theorem, we now proved

$$
\left.\left.e_{\mathrm{el}, \varepsilon}\right|_{Q_{\mathrm{S}}} \rightarrow e_{\mathrm{el}}\right|_{Q_{\mathrm{S}}} \quad \text { strongly in } L^{2}\left(Q_{\mathrm{S}} ; \mathbb{R}_{\mathrm{sym}}^{3 \times 3}\right),
$$

which is to be used for the limit passage in the nonlinear term $\partial_{e} \mathcal{G}_{\mathrm{E}}$.

The energy conservation now holds due to the proved regularity, as $\sqrt{\varrho} \ddot{u} \in L^{\infty}\left(I ; L^{2}\left(\Omega ; \mathbb{R}^{3}\right)\right)$ is surely in duality with $\sqrt{\varrho} \dot{u} \in W^{1, \infty}\left(I ; L^{2}\left(\Omega ; \mathbb{R}^{3}\right)\right)$, cf. (5.21a). Eventually, like we did in (5.17), we now can show that

$$
\begin{aligned}
& \limsup _{\varepsilon \rightarrow 0} \int_{Q_{\mathrm{F}}} \varepsilon \mathbb{D}_{\mathrm{KV}} e\left(\dot{u}_{\varepsilon}\right): e\left(\dot{u}_{\varepsilon}\right) \mathrm{d} x \mathrm{~d} t \leq \int_{\mathbb{R}^{3}} \varrho_{\mathrm{ext}}(T) \phi(T)-\frac{1}{8 \pi g}|\nabla \phi(T)|^{2} \mathrm{~d} x \\
& -\int_{\Omega} \varrho \nabla \phi(T) \cdot u(T)-\varrho \phi(T) \mathrm{d} x-\int_{0}^{T} \int_{\mathbb{R}^{3}} \dot{\varrho}_{\mathrm{ext}} \phi \mathrm{d} x \mathrm{~d} t-\int_{Q}(\omega \times(\omega \times(x+u) \cdot \dot{u} \mathrm{~d} x \mathrm{~d} t \\
& \quad-\int_{\Omega} \frac{\varrho}{2}|\dot{u}(T)|^{2}+\frac{3}{2} K_{\mathrm{E}}\left|\operatorname{sph} e_{\mathrm{el}}(T)\right|^{2} \mathrm{~d} x-\int_{\Omega_{\mathrm{S}}} \mathcal{G}_{\mathrm{E}}\left(\alpha(T), e_{\mathrm{el}}(T)\right)+\frac{\kappa}{2}|\nabla \alpha(T)|^{2} \mathrm{~d} x \\
& \quad-\int_{Q_{\mathrm{S}}} \mathbb{D}_{\mathrm{KV}} \dot{e}_{\mathrm{el}}: \dot{e}_{\mathrm{el}}+\mathbb{D}_{\mathrm{MX}} \dot{\pi}: \dot{\pi}+\dot{\alpha} \eta^{\prime}(\dot{\alpha}) \mathrm{d} x \mathrm{~d} t+E_{0}=0,
\end{aligned}
$$

with $E_{0}$ from (5.5). The last equality is due to the mentioned energy conservation. Thus (4.3) is proved.

Remark 5.6 (The successive convergence). Note that, in (5.19), we benefited from having $G_{\mathrm{E}}$ already pushed to zero on $\Omega_{\mathrm{F}}$ because the viscosity on $\Omega_{\mathrm{F}}$ is (intentionally) not uniformly controlled, being limited to zero. Thus the direct merging of both limit processes in Propositions 3.1 and 4.1 is not possible. Of course, a suitable scaling between these two would facilitate such connection.

Remark 5.7 (Discontinuities in $\varrho$ accros the Earth interfaces). The interfaces between the ocean beds and the mantle as well as the Gutenberg's discontinuity and between the inner and outer core regions typically also exhibit discontinuities in mass density $\varrho$, which is incompatible with the assumption $\varrho \in W^{1,3}(\Omega)$ in (5.1a) used in (5.10). Note that the additional regularity of the initial conditions involved in Definition 5.5 allowed us to avoid this restriction and consider a general, possibly discontinuous $\varrho \in L^{\infty}(\Omega)$. A respective modification of the proofs of Propositions 2.1 3.1 would be possible, too. 
Acknowledgments. The author is thankful to Katharina Brazda and Ctirad Matyska for many fruitful discussions about the models. Moreover, deep thanks are also for hospitality and support of the Erwin Schrödinger Institute, Univ. Vienna, and for the partial support of the Czech Science Foundation projects 16-03823S and 17-04301S and also by the Austrian-Czech projects 16-34894L (FWF/CSF), as well as through the institutional support RVO: 61388998 (ČR).

\section{References}

[1] Y. Ben-Zion. Dynamic ruptures in recent models of earthquake faults. J. Mech. Phys. Solids, 49:2209-2244, 2001.

[2] Y. Ben-Zion and J.-P. Ampuero. Seismic radiation from regions sustaining material damage. Geophys. J. Int., 178:1351-1356, 2009.

[3] D. Boger. A highly elastic constant-viscosity fluid. J. Non-Newtonian Fluid Mechanics, 3:87-91, 1977.

[4] K. Brazda. The elastic-gravitational equations in global seismology with low regularity. $\mathrm{PhD}$ thesis, Univ. Wien, 2017.

[5] K. Brazda, M. V. de Hoop, and G. Hoermann. Variational formulation of the earth's elasticgravitational deformations under low regularity conditions. Preprint arXiv:1702.04741, 2017.

[6] F. A. Dahlen and J. Tromp. Theoretical global seismology. Princetown Univ. Press, Princetown, NJ, 1998.

[7] A. Green and P. Naghdi. A general theory of an elastic-plastic continuum. Arch. Rational Mech. Anal., 18:251-281, 1965.

[8] R. A. Harris et al. The SCEC/USGS dynamic earthquake rupture code verification exercise. Seismological Res. Lett., 80:119-126, 2009.

[9] Y. Huang, J.-P. Ampuero, and D. V. Helmberger. Earthquake ruptures modulated by waves in damaged fault zones. J. of Geophysical Research: Solid Earth, B9:3133-3154, 2014.

[10] Y. Kaneko, N. Lapusta, and J.-P. Ampuero. Spectral element modeling of spontaneous earthquake rupture on rate and state faults: Effect of velocity-strengthening friction at shallow depths. J. Geophysical Res., 113:B09317, 2008.

[11] D. Komatitsch and J. Tromp. Spectral-element simulations of global seismic wave propagation - I. validation. Geophys. J. Int., 149:390-412, 2002.

[12] D. Komatitsch and J. Tromp. Spectral-element simulations of global seismic wave propagation - II. three-dimensional models, oceans, rotation and self-gravitation. Geophys. J. Int., 150:303-318, 2002.

[13] L. Koot and M. Dumberry. Viscosity of the Earth's inner core: Constraints from nutation observations. Earth and Planetary Science Letters, 308(3):343-349, 2011.

[14] T. Lay and T. C. Wallace. Modern global seismology. Acad. Press, San Diego, 1995.

[15] V. Lyakhovsky and Y. Ben-Zion. Damage-breakage rheology model and solid-granular transition near brittle instability. J. Mech. Phys. Solids, 64:184-197, 2014.

[16] V. Lyakhovsky, Y. Hamiel, J.-P. Ampuero, and Y. Ben-Zion. Non-linear damage rheology and wave resonance in rocks. Geophys. J. Int., 178:910-920, 2009.

[17] V. Lyakhovsky, Y. Hamiel, and Y. Ben-Zion. A non-local visco-elastic damage model and dynamic fracturing. J. Mech. Phys. Solids, 59:1752-1776, 2011. 
[18] V. Lyakhovsky and V. P. Myasnikov. On the behavior of elastic cracked solid. Phys. Solid Earth, 10:71-75, 1984.

[19] T. Maedae and T. Furumura. FDM simulation of seismic waves, ocean acoustic waves, and tsunamis based on tsunami-coupled equations of motion. Pure Appl. Geophys., 170:109 $127,2013$.

[20] C. Pelties, J. de la Puente, J.-P. Ampuero, G. B. Brietzke, and M. Käser. Three-dimensional dynamic rupture simulation with a high-order discontinuous galerkin method on unstructured tetrahedral meshes. J. Geophys. Res., 117:B02309, 2012.

[21] K. R. Rajagopal and T. Roubíček. On the effect of dissipation in shape-memory alloys. Nonlinear Anal., Real World Appl., 4:581-597, 2003.

[22] T. Roubíček. Nonlinear Partial Differential Equations with Applications. Birkhäuser, Basel, 2nd edition, 2013.

[23] T. Roubíček. A note about the rate-and-state-dependent friction model in a thermodynamical framework of the Biot-type equation. Geophysical J. Intl., 199:286-295, 2014.

[24] T. Roubíček. Geophysical models of heat and fluid flow in damageable poro-elastic continua. Cont. Mech. Thermodyn., 29:625-646, 2017.

[25] T. Roubíček, C. G. Panagiotopoulos, and V. Mantič. Quasistatic adhesive contact of viscoelastic bodies and its numerical treatment for very small viscosity. Zeitschrift angew. Math. Mech., 93:823-840, 2013.

[26] T. Roubíček, O. Souček, and R. Vodička. A model of rupturing lithospheric faults with re-occurring earthquakes. SIAM J. Appl. Math., 73:1460-1488, 2013.

[27] R. A. Secco. Viscosity of the outer core. In T. Ahrens, editor, Mineral Physics \& Crystallography: A Handbook of Physical Constants, pages 218-226. Willey, 2013.

[28] D. E. Smylie and A. Palmer. Viscosity of Earth's outer core. Preprint arXiv:0709.3333, 2007.

[29] N. Tosi, O. Čadek, and Z. Martinec. Subducted slabs and lateral viscosity variations: effects on the long-wavelength geoid. Geophys. J. Int., 179:813-826, 2009.

[30] V. Tsai, J.-P. Ampuero, H. Kanamori, and D. Stevenson. Estimating the effect of Earth elasticity and variable water density on tsunami speeds. Geophysical Res. Letters, 40:492496, 2013.

[31] G. A. D. Wijs, G. Kresse, L. Vočadlo, D. Dobson, D. Alfé, M. J. Gillan, and G. D. Price. The viscosity of liquid iron at the physical conditions of the Earth's core. Nature, 392 (6678):805-807, 1998.

[32] J. H. Woodhouse and A. Deuss. Theory and observations - Earth's free oscillations. In B. Romanowicz and A. Dziewonski, editors, Seismology and Structure of the Earth: Treatise on Geophysics, volume 1, chapter 1.02, pages 31-65. Elsevier, 2009. 